This is the accepted version of the following article:

Ribes-Guardiola, P., Poy, R., Patrick, C. J., \& Moltó, J. (2020). Electrocortical Measures of Performance Monitoring from Go/No-Go and Flanker Tasks: Differential Relations with Trait Dimensions of the Triarchic Model of Psychopathy. DOI: 10.1111/psyp.1357, which has been published in final form at [https://onlinelibrary.wiley.com/doi/abs/10.1111/psyp.13573]

This article may be used for non-commercial purposes in accordance with the Wiley Self-Archiving Policy [http://www.wileyauthors.com/selfarchiving]. 
Performance Monitoring ERPs and Triarchic Psychopathy

Running Head: Performance Monitoring ERPs and Triarchic Psychopathy

\section{Electrocortical Measures of Performance Monitoring from Go/No-Go and Flanker Tasks: Differential Relations with Trait Dimensions of the Triarchic Model of Psychopathy}

Pablo Ribes-Guardiola ${ }^{\mathrm{a}}$, Rosario Poy ${ }^{\mathrm{a}}$, Christopher J. Patrick ${ }^{\mathrm{b}}, \&$ Javier Moltó $^{\mathrm{a}}$

${ }^{a}$ Affective Neuroscience Lab, Department of Basic and Clinical Psychology, and Psychobiology. Universitat Jaume I, Avenida Sos Baynat s/n 12071, Castelló, Spain

${ }^{b}$ Department of Psychology, Florida State University, 1107 West Call Street, Tallahassee, FL 32306, United States

Address correspondence to:

Pablo Ribes-Guardiola, $\mathrm{PhD}$

Department of Basic and Clinical Psychology, and Psychobiology

Universitat Jaume I Avenida Sos Baynat s/n 12071, Castellón, Spain.

Phone: +34964729990

Email:pribes@uji.es

Javier Moltó, $\mathrm{PhD}$

Department of Basic and Clinical Psychology, and Psychobiology

Universitat Jaume I Avenida Sos Baynat s/n 12071, Castellón, Spain.

Phone: +34964729581

Email: molto@uji.es 


\begin{abstract}
This study examined associations of performance-monitoring ERPs from go/no-go and flanker tasks with one another, and with psychopathy-related traits of disinhibition, meanness, and boldness. A task-dependent relationship was evident between the errorrelated negativity (ERN) and trait disinhibition, with high-disinhibited participants showing reduced no-go ERN but not flanker ERN. Disinhibition was also inversely related to variants of the $\mathrm{P} 3$ and the error positivity (Pe) from these two tasks. A factor analysis of the ERPs revealed two distinct factors, one reflecting shared variance among the P3 and Pe measures from the two tasks, and the other covariance among the N2 and ERN measures. Scores on the P3/Pe factor, but not the N2/ERN factor, were inversely related to disinhibition, and accounted for associations of this trait with variants of the $\mathrm{P} 3$ and Pe across tasks. The implication is that high trait disinhibition relates mainly to reductions in brain responses associated with later elaborative stages in the processing of motivational significant events across different tasks. Importantly, no-go ERN predicted disinhibition scores beyond N2/ERN factor scores, indicating that high disinhibition is not generally related to diminished early preresponse conflict and error processing, but rather to processing impairments in conditions calling for inhibition of prepotent response tendencies.
\end{abstract}

Keywords: Disinhibition, performance monitoring, Event-Related Potentials, go/no-go task, flanker task 
Performance Monitoring ERPs and Triarchic Psychopathy

\section{Introduction}

Psychopathy is a multifaceted personality disorder encompassing distinct clusters of affective, interpersonal, and behavioral deviance features (Cleckley, 1941/1976; Hare, 2003). Despite ongoing debates regarding the nature, core elements, and boundaries of this disorder (Skeem, Polaschek, Patrick, \& Lilienfeld, 2011), behavioral deviance features such as poor judgment and failure to learn by experience, poor behavioral controls, impulsivity, and a lack of planfulness are present in all conceptions of psychopathy and instruments for assessing it (e.g., Cleckley, 1976; Hare, 2003; Lilienfeld \& Widows, 2005; Patrick, Fowles, \& Krueger, 2009). Consequently, the study of deviations in the brain mechanisms underlying performance monitoring and the adaptive control of behavior in samples varying in psychopathic traits may help to clarify and delineate the specific neurocognitive processes that underlie certain symptomatic features of psychopathy (see Patrick, 2018, for a recent review).

Over the past two decades, event-related potentials (ERPs) derived from electroencephalographic (EEG) recordings have been used to characterize the neural mechanisms and temporal dynamics of performance monitoring at different stages of goal-directed behavior, from stimulus processing and action selection to error and feedback processing (see Ullsperger, Fischer, Nigbur, \& Endrass, 2014, for a review). Much of this research has employed diverse speeded response conflict tasks, such as go/no-go and flanker discrimination tasks, to study the ERP correlates of important cognitive control processes, including error monitoring, interference control, and inhibitory processing. In brief, during action selection - when participants must withhold a prepotent 'go' response on rarely occurring 'no-go' trials of an action/inhibition (go/nogo) task, or suppress the interference produced by distracting stimuli surrounding a central target on incongruent trials relative to congruent trials of a flanker task (Eriksen \& 
Eriksen, 1974) - a frontocentral negative ERP deflection, the N2, can be observed between 250-350 ms after stimulus onset (see Folstein \& van Petten, 2008, for a review). The N2 is typically more negative on incongruent compared to congruent stimulus trials in variants of the flanker task (Kopp, Rist, \& Mattler, 1996; van Veen \& Carter, 2002), as well as on no-go versus go trials of go/no-go tasks (see Huster, Enriquez-Geppert, Lavallee, Falkenstein, \& Herrmann, 2013 for a review), and its functional significance in these paradigms has been linked to inhibitory (Falkenstein, Hoormann, \& Hohnsbein, 1999; Jodo \& Kayama, 1992; Kopp, Mattler, Goertz, \& Rist, 1996) or response conflictmonitoring processes (Nieuwenhuis, Yeung, van den Wildenger, \& Ridderinkhof, 2003; Yeung, Botvinick, \& Cohen, 2004). The N2 is followed by the P3, a positive ERP deflection that peaks over central and parietal sites between 300 and $600 \mathrm{~ms}$ following stimulus onset; this component has been functionally linked to attentional resource allocation, memory and context updating, and evaluative or elaborative processing of motivationally significant or otherwise salient events (Donchin \& Coles, 1988; Kok, 2001; Nieuwenhuis, Aston-Jones, \& Cohen, 2005; Polich, 2007). In go/no-go tasks, enhanced P3 on no-go trials has been interpreted as indexing cognitive processes supporting response inhibition, or as reflecting evaluative processing stages of the response inhibition process or its outcome (e.g., Albert, López-Marín, Hinojosa, \& Carretié, 2013; see Huster et al., 2013 for a review).

Both of these tasks, go/no-go and flanker, have also been widely employed in the study of error processing. Following response inhibition errors or action slips due to interference, the error-locked ERP exhibits a sharp, early (maximal within the first $100 \mathrm{~ms}$ after errors) frontocentral negative-polarity deflection, termed the error-related negativity (ERN; Falkenstein, Hohnsbein, Hoormann, \& Blanke, 1991; Gehring, Goss, Coles, Meyer, \& Donchin, 1993) that is followed by a more sustained centroparietal positive 
deflection, termed the error positivity (Pe), that peaks between 200 and $500 \mathrm{~ms}$ after errors (Falkenstein, Hoormann, Christ, \& Hohnsbein, 2000). Although the functional significance of both components is still under debate (Gehring, Liu, Orr, \& Carp, 2012; Overveek, Nieuwenhuis, \& Ridderinkhof, 2005, for reviews), the ERN is thought to represent early and relatively automatic stages of error processing, and has been conceptualized as a mismatch/error detection signal (Coles, Scheffers, \& Holroyd, 2001; Falkenstein et al., 2000; Gehring et al., 1993), a negative reinforcement learning signal (Holroyd \& Coles, 2002), or as a marker of postresponse conflict processing (Yeung et al., 2004). The Pe, on the other hand, is believed to reflect more elaborated stages of error processing, such as conscious error recognition (Nieuwhenhuis, Ridderinkhof, Blom, Band, \& Kok, 2001; O’Connell et al., 2007), affective appraisal of error significance (Falkenstein et al., 2000; van Veen \& Carter, 2002), or a P3-like response related to the perceptual salience or motivational significance of error commission (Arbel \& Donchin,

2009; Leuthold \& Sommer, 1999; Overbeek et al., 2005; Ridderinkhof, Ramautar, \& Wijnen, 2009).

The current study was undertaken to investigate how variants of the aforementioned ERP components - stimulus N2 and P3, and error-response ERN and Pe from both go/no-go and flanker tasks relate to different trait dimensions of psychopathy as described in the triarchic model of psychopathy (Patrick et al., 2009). Our major hypotheses for this family of ERP responses focused on the impulsive-unrestrained (i.e., disinhibitory) dimension of the triarchic model.

\subsection{The triarchic model of psychopathy and externalizing proneness}

The triarchic model was formulated as a framework for reconciling alternative conceptions of psychopathy and helping to guide research on neurobiological and developmental processes contributing to this disorder (Patrick et al., 2009). According to 
this model, different conceptualizations of psychopathy place varying degrees of emphasis on three distinct dispositional constructs: disinhibition (involving nonplanfulness, irresponsibility, impaired regulation of emotions and urges and deficient behavioral restraint), meanness (entailing deficient empathy, lack of close attachments with others, callousness, exploitativeness, and empowerment through cruelty), and boldness (encompassing high social dominance, tolerance for risk and uncertainty, and the ability to remain calm under threat and recover rapidly from stressors). A key aspect of the model is that the constructs of disinhibition, meanness, and boldness correspond to trait constructs with clear neurobiological and developmental referents (for a recent conceptual review, see Patrick \& Drislane, 2015).

Consistent with this perspective, increasing empirical evidence has accumulated for diverging relations of the affective/interpersonal and impulsive/antisocial symptom components of psychopathy with criterion measures in different modalities, including psychophysiological measures - leading to the idea that these two symptom components are undergirded by different neurobehavioral mechanisms (Fowles \& Dindo, 2006, 2009; Patrick \& Bernat, 2009). A neurobehavioral dimension of trait fearlessness is considered more pertinent to understanding the affective/interpersonal (Factor 1) features of psychopathy, represented by boldness and meanness dimensions in the triarchic model (Patrick et al., 2009; Skeem et al. 2011). On the other hand a neurobehavioral dimension of externalizing proneness, reflecting dispositional liability toward a range of impulse control disorders (Krueger et al., 2002; Krueger, Markon, Patrick, Benning, \& Kramer, 2007), is considered more relevant to the impulsive/antisocial (Factor 2) features of psychopathy (Patrick, Hicks, Krueger, \& Lang, 2005).

From a neurobiological standpoint, externalizing proneness is theorized to reflect impairments in the functioning of frontal brain systems implicated in the regulation of 
behavioral and affective reactions (Patrick \& Bernat, 2009; see also Patrick, Durbin, \& Moser, 2012, for reviews). The best-established ERP indicator of externalizing proneness is a reduced amplitude of the visually evoked P3 response (Euser et al., 2012; Gao \& Raine, 2009; Iacono, Malone, \& McGue, 2003), a relationship that has been evidenced across a variety of tasks, including oddball (Nelson, Patrick, \& Bernat, 2011; Patrick et al., 2006; Patrick et al., 2013; Venables et al., 2018), gambling (Bernat, Nelson, Steele, Gehring, \& Patrick, 2011) and picture viewing tasks (Perkins et al., 2017), along with flanker tasks (Nelson et al., 2011; Venables et al., 2018), and modified oddball/no-go tasks (Brennan \& Baskin-Sommers, 2018). In addition to P3 amplitude reduction, higher levels of externalizing proneness have also been linked to deficits in laboratory performance measures of inhibitory control (Venables et al., 2018; Young et al., 2009), and to reduced amplitude of ERN response (Hall, Bernat, \& Patrick, 2007).

From a triarchic model perspective, the disinhibition dimension represents a counterpart to the externalizing liability factor (see Patrick, 2018) and, as such, should show selective relations with ERP correlates of performance monitoring linked to externalizing proneness. In the next section we provide a comparative review of the rather inconsistent literature on performance monitoring deficits in psychopathy, with reference to known ERP correlates of externalizing proneness cited in dual-process models of psychopathy (Patrick \& Bernat, 2009).

\subsection{Performance monitoring deficits in psychopathy and externalizing}

\section{psychopathology}

Studies addressing ERP correlates of performance monitoring during action selection in interference and inhibitory paradigms, which are few in number, have been dominated by unitary conceptualizations of psychopathy. On one hand, no published study has yet examined variations in N2 and P3 amplitudes during flanker tasks in 
samples assessed for psychopathic traits, and evidence for impaired response inhibition processing in go/no-go tasks is mixed, with some studies showing diminished no-go N2 (Kiehl, Smith, Hare, \& Liddle, 2000), but others showing instead reduced no-go P3 (Kim \& Jung, 2014), and yet others demonstrating nonaltered no-go N2 nor P3 amplitudes in high-psychopathic individuals (Maurer, Steele, Edwards, et al., 2016; Munro et al., 2007a). By contrast, P3 amplitude reduction has been found in relation to externalizing problems and traits in both flanker discrimination and inhibitory (stop-signal; modified oddball/no-go) tasks (Brennan \& Baskin-Sommers, 2018; Nelson et al., 2011; Venables et al., 2018; Vilà-Balló, Hdez-Lafuente, Rostan, Cunillera, \& Rodríguez-Fornells, 2014).

Although more extensive, research on error processing in psychopathy has yielded conflicting results as well. Studies of incarcerated offenders assessed using the Psychopathy Checklist-Revised (PCL-R; Hare, 2003) or its derivatives suggest a general pattern of intact ERN amplitude during standard (cognitive) speeded response tasks (e.g., Brazil et al., 2009; Maurer, Steele, Cope, et al., 2016; Maurer, Steele, Edwards, et al., 2016; Munro et al., 2007b; Steele, Maurer, Bernat, Calhoun, \& Kiehl, 2016), whereas studies with community samples employing self-report assessment instruments - such as the Psychopathic Personality Inventory (PPI; Lilienfeld \& Widows, 2005) or the Triarchic Psychopathy Measure (TriPM; Patrick, 2010) - have reported diminished ERN amplitude in relation to higher scores on disinhibition and impulsive-antisocial traits in different tasks (Heritage \& Benning, 2013; Pasion, Cruz, \& Barbosa, 2016). Bresin, Finy, Sprague, and Verona (2014) likewise reported evidence for reduced ERN in relation to impulsiveantisocial features in a sample assessed for psychopathy using the screening version of Hare's PCL-R.

More recently, however, Venables et al. (2018) did not find evidence for a relationship between the ERN measured in an "arrow" variant of the flanker task and 
Performance Monitoring ERPs and Triarchic Psychopathy

scale measures of externalizing proneness, suggesting that the relationship between the ERN and externalizing (disinhibition) may be task-dependent (see Pasion \& Barbosa, 2019, for meta-analytic evidence showing stronger associations between no-go variants of the ERN response and externalizing). Finally, and with regard to later stages of error processing - as indexed by the $\mathrm{Pe}$ - results from studies conducted in incarcerated populations have yielded mixed findings (see Brazil et al., 2009; Maurer, Steele, Cope, et al., 2016; Maurer, Steele, Edwards, et al., 2016; Munro et al., 2007b; Steele et al., 2016), and this ERP component has not been the focus of past error-processing studies conducted in community adults (Heritage \& Benning, 2013; Pasion et al., 2016). Of note, however, Venables et al. (2018) found that flanker-task Pe overlapped with stimulus-P3 measures from other tasks, which covaried in turn with scale measures of disinhibition, suggesting the possibility that reduced Pe amplitude taps an externalizing-related brain process in common with other variants of $\mathrm{P} 3$ (Venables et al., 2018). However, this finding requires corroboration, and the specificity of this association to disinhibition (vis-à-vis other triarchic trait dimensions) remains to be determined.

From the evidence reviewed, heterogeneity across past studies in how psychopathy is conceptualized (i.e., unitary vs. multifaceted construct perspective on psychopathy; see Skeem et al., 2011) and operationalized (e.g., interview-based vs. self-report assessment instruments) can be regarded as potential sources of inconsistencies, to the extent that different psychopathy measures used in prior studies emphasize impulsive-externalizing (disinhibitory) as opposed to affective-interpersonal (bold, mean) features (for detailed reviews, see: Pasion, Fernandes, Pereira, \& Barbosa, 2018; Schulreich, 2016).

\subsection{Current study aims and hypotheses}

In light of the foregoing evidence, there is a clear need to further investigate abnormalities in ERP indices of performance monitoring in samples varying in 
psychopathic traits. Rather than focusing on one single monitoring process or brain-ERP indicator from a particular performance task, the current study undertook to characterize, in a relatively large sample of undergraduates, associations for ERP indicators from tasks designed to invoke distinct processes - interference control (flanker N2 and flanker P3), response inhibition (no-go N2 and no-go P3), and error monitoring (ERN and Pe) - with the three dispositional dimensions of the triarchic model of psychopathy (Patrick et al., 2009). In particular, we (1) examined the differential contributions of disinhibition, meanness, and boldness dimensions to these performance monitoring-related ERPs, and (2) tested for a possible moderating role of the task context (i.e., go/no-go vs. flanker) in such associations.

This latter point is important in light of research demonstrating varying levels of cross-task correlations among diverse performance monitoring ERPs. For example, research on error processing points to varying degrees of reliability (Meyer, Riesel, \& Hajcak, 2013; Riesel, Weinberg, Endrass, Meyer, \& Hajcak, 2013) and convergence among ERN (range $=.33-.43)$ and Pe (range $=.37-.49)$ measures from different tasks (Riesel et al., 2013), suggesting the presence of both shared and task specific variance, which can have important implications for psychopathology and individual differences studies (see Weinberg, Dieterich, \& Riesel, 2015, for a review). Along with this, some studies have reported positive correlations between P3 and Pe both within and across tasks (Burwell, Malone, \& Iacono, 2016; Cassidy, Robertson, \& O’Conell, 2012; Davies, Segalowitz, Dywan, \& Pailing, 2001), as well as between ERN and N2 (Amodio, Master, Yee, \& Taylor, 2008; Gruendler, Ullsperger, \& Huster, 2011; Yeung et al., 2004). Hence, systematic examination of the reliability and convergent and discriminant validity of neural response variables across tasks is important for clarifying their utility as individual difference measures (Hajcak, Meyer, \& Kotov, 2017; Patrick \& Hajcak, 2016). 
Performance Monitoring ERPs and Triarchic Psychopathy

Of particular relevance to the current study, considerable progress has been made toward operationalizing the dispositional construct of externalizing proneness (disinhibition) in terms of factors reflecting the shared variance among different ERP and self-report indicators (i.e., psychoneurometric factors; see Nelson et al., 2011; Patrick et al., 2013; Venables et al., 2018). This multi-method approach to quantifying dispositional characteristics provides a means to enhance the prediction of criterion measures of interest from different assessment modalities. Strong evidence exists for convergence among different variants of the P3 and mutual overlap with scale-assessed disinhibition (Nelson et al., 2011; Patrick et al., 2013; Venables et al., 2018), but evidence for convergence of other neural indices of performance-monitoring - including the N2, ERN, and Pe - is less clear. With this in mind, the current study tested for covariance among various performance-monitoring ERP indicators across go/no-go and flanker tasks in order to clarify their overlap versus uniqueness as indicators of neural processes related to one or more of the triarchic constructs (e.g., Patrick \& Hajcak, 2016).

Based on the above-reviewed evidence, and drawing on ideas put forth by dual process models of psychopathy (Fowles \& Dindo, 2006, 2009; Patrick \& Bernat, 2009), major hypotheses of the current study were as follows:

(1) On the basis of prior work demonstrating reductions in different variants of stimulus-locked P3 for individuals high in externalizing problems and traits (e.g., Brennan \& Baskin-Sommers, 2018; Nelson et al., 2011; Patrick et al., 2013; Perkins et al., 2017; Venables et al., 2018), we predicted that scores on the triarchic dimension of disinhibition would show significant negative associations with variants of stimulus P3 from each of our tasks (go/no-go, flanker).

(2) We predicted that higher disinhibition scores would be also associated with a reduced amplitude of ERN response — particularly in the go/no-go task (see Pasion \& 
Barbosa, 2019 for a meta-analysis), as previously demonstrated for participants scoring high in externalizing proneness (Hall et al., 2007) and in relation to impulsive/antisocial features of psychopathy in noncriminal samples (Heritage \& Benning, 2013; Pasion et al., 2016).

(3) With regard to the Pe, there are inconsistencies as noted earlier in findings for offender samples, and little research has examined relations of this ERP measure with psychopathy symptom dimensions. On the other hand, converging lines of evidence support a link between this ERP component and stimulus-P3 variants known to covary with externalizing proneness (e.g., Burwell et al., 2016; Venables et al., 2018). Accordingly, we predicted that (a) Pe responses from the go/no-go and flanker tasks would covary with stimulus-P3 responses from these tasks, and more tentatively, (b) Pe like stimulus P3 - would show a negative association with triarchic disinhibition (i.e., smaller amplitude of response for participants scoring high as compared to low in disinhibition).

Finally, although some evidence has been reported for reduced inhibition-related N2 amplitude in high-psychopathic adult offenders (Kiehl et al., 2000) and violent juvenile offenders (Vilà-Balló et al., 2014), limited grounds exist for advancing specific hypotheses regarding associations of this ERP measure with the three triarchic dimensions in the current, nonoffender sample. Our analyses for this measure were thus exploratory in nature.

\section{Method}

\subsection{Participants}

A total of 161 undergraduate students ( 114 women, 47 men; $M$ age $=20.55$ years, $S D=4.51)$ from the Universitat Jaume I of Castellón (Spain) were contacted and agreed to participate in a laboratory assessment procedure for course credit. No participants 
evidenced noncorrected visual or auditory impairments or were undergoing psychiatric or pharmacological treatment at the time of testing. All participants were informed about the nature of the study and provided informed consent. The Spanish adaptation (Esteller, Poy, \& Moltó, 2016; Poy, Segarra, Esteller, López, \& Moltó, 2014) of the Triarchic Psychopathy Measure (TriPM; Patrick, 2010) was used to assess psychopathic traits. This measure was developed to provide a comprehensive assessment of the three dispositional dimensions of boldness, meanness, and disinhibition represented in the triarchic model of psychopathy (Patrick et al., 2009). Its 58 items are answered using a 4-point Likert response format ( $0=$ "false"; 1 = "somewhat false"; 2 = "somewhat true"; 3 = "true"). Scale scores can thus range from 0 - 57 for the 19-item Boldness and Meanness scales, and from $0-60$ for the 20 -item Disinhibition scale. In current the study sample, Cronbach's alpha coefficients for TriPM Boldness, Meanness, and Disinhibition scores were $0.81,0.81$, and 0.80 , respectively. Descriptive statistics for TriPM scale scores for the sample as a whole, and for women and men separately, are provided in the Supplementary Materials; also included in the Supplement are independent samples $t$-tests for significant gender differences on TriPM scale scores, and evidence for the construct validity of the three TriPM scale scores in the current sample.

From the initial sample of 161 participants, 7 were excluded from analyses due to: discontinuation of participation in the experimental session $(n=1)$, equipment malfunction preventing the storage of behavioral data $(n=1)$, or anomalously poor performance on either the go/no-go task (1 participant who failed to follow task instructions, responding to no-go rather than to go stimuli) or the arrow flanker task (4 participants with overall accuracy below the $75 \%$ required by task instructions; see below). Of the remaining participants, 12 were excluded from further analyses due to an excessive number of artifacts and/or having less than 6 artifact-free trials to aggregate into 
error-related ERPs (cf. Olvet \& Hajcak, 2009). Given our interest in examining covariance between the different stimulus and response-locked ERP measures, the results we report are based on those participants who produced valid behavioral and ERP data for both tasks $(n=142 ; 101$ women, $M$ age $=20.58, S D=4.69)$.

\subsection{Tasks and procedure}

Experimental testing was conducted individually in an isolated and dimly lit room where participants were seated $110 \mathrm{~cm}$ from the monitor screen on which stimuli were displayed. Prior to testing, participants provided informed consent. Participants performed both a response inhibition go/no-go task and an arrow version (Hajcak, Moser, Yeung, \& Simons, 2005; Olvet \& Hajcak, 2009) of the flanker task. Task order was counterbalanced across participants. Participants were instructed to respond both as quickly and as accurately as possible in both tasks. A PC Pentium Core 2 Duo (Intel) computer running Presentation ${ }^{\circledR}$ v.14.5 software (Neurobehavioral Systems, Inc. Albany, Ca, USA) was used to control the order and timing of stimulus presentations and to record behavioral responses, which were performed using an SRBox 200A serial response device (EGI). The duration of the testing session was about $2 \mathrm{hr}$.

\subsubsection{Go/no-go task}

On each trial of the go/no-go task (described by Nieuwenhuis et al., 2003) either the letter "M" or "W" - presented in white, Arial size-50 font, subtending $0.5^{\circ}$ vertically and $0.7^{\circ}$ horizontally - appeared below a white fixation dot which remained in the center of the screen against a black background for the full 100-ms duration of the trial. The intervals between successive stimuli were one of five equiprobable durations of 1100 , $1300,1500,1700$ or $1900 \mathrm{~ms}$. Participants were instructed to respond (button press with their dominant index finger) to the go stimuli and withhold responding to the no-go stimuli. Letter assignment to the go and no-go condition was counterbalanced across 
participants. The task included 1200 trials, divided into 6 blocks of 200, between which participants had 1-min rest breaks. To ensure a dominant go response and to enhance the difficulty of withholding responses to no-go stimuli, the frequency of go and no-go trials was $80 \%$ and $20 \%$, respectively. This frequency distribution resulted in a total of 960 go trials -160 per block - and a total of 240 no-go trials -40 per block. Go and no-go trial types were presented in a pseudo-random order that precluded the consecutive presentation of two no-go trials. Before starting the task, participants performed 50 practice trials in which go and no-go stimuli each appeared $50 \%$ of the time. The overall duration of the task, including the practice trials and breaks, was about $40 \mathrm{~min}$.

\subsubsection{Arrow flanker task}

A variant of the flanker task (Eriksen \& Eriksen, 1974) was used in the current study (see Hajcak et al., 2005; Olvet \& Hajcak, 2009). On each task trial, a string of five arrows was presented horizontally in the center of the screen (") $>>>>>$ ", "<<<<<", " $>><>>$ ", or " $<<><<")$ - in white, Arial size-50 font, subtending $1.3^{\circ}$ vertically and $9.2^{\circ}$ horizontally - for a duration of $200 \mathrm{~ms}$. A fixation cross $(+)$ appeared for $150 \mathrm{~ms}$ preceding the onset of each arrow-string stimulus. The intervals between consecutive stimuli, including the fixation cross, were of six equiprobable durations of 1500, 1600, $1700,1800,1900$ or $2000 \mathrm{~ms}$. Participants were instructed to attend to the central arrow of the array and make a left or right index-finger button press according to the direction left $(<)$ or right $(>)$ - of the central arrow. The task consisted of 576 trials, divided into 12 blocks of 48 trials. A total of 144 stimuli of each four types were presented - congruent left $(<<<<<)$, congruent right $(>>>>>)$, incongruent left $(>><>>)$, and incongruent right $(<<<<)-$ resulting in 12 stimuli of each type per block and a frequency of $50 \%$ of congruent and incongruent trials. Stimuli were presented in random order. After each block, participants received three types of feedback instructing them to adapt their 
response style according to their performance: "Please, try to respond faster" (for performance accuracy $>90 \%$ ); "Please, try to be more accurate" (for performance accuracy $<75 \%$ ); and "You are doing a great job" (for performance accuracy between $75 \%$ and $90 \%$ ). Feedback messages were presented on the screen for $6 \mathrm{~s}$, after which participants had a self-paced break before starting the next trial block. Prior to performing the actual task, participants completed 48 practice trials that included equal frequencies $(50 \%)$ of congruent and incongruent trials. The total duration of the task, including the practice trials and breaks, was about $25 \mathrm{~min}$.

\subsection{Psychophysiological Recording and Data Reduction}

EEG activity was recorded from 256 electrodes using HydroCel Geodesic Sensor Nets (HCGSN) and amplified and filtered (analog filters: 0.10-100 Hz bandpass) using an Electrical Geodesics, Inc. (EGI; Oregon, USA) NetAmps 300 amplifier system. The software NetStation v.4.4.2 (EGI), installed on a MacBook Pro (Apple) computer, was used to record and store the continuous EEG signal, as well as the digital TTL signals used to index stimulus timing and response execution, which were received through a parallel AV Device DIN Adapter (EGI). During acquisition of signal data, EEG was referenced to the vertex scalp site $(\mathrm{Cz})$ and digitized continuously at a sampling rate of $250 \mathrm{~Hz}$ with a 24-bit analog-to-digital converter. Scalp impedances were kept below 50 $\mathrm{k} \Omega$, as recommended by manufacturer guidelines.

Preprocessing of the raw EEG data was performed offline using Brain Electrical Source Analysis software (BESA v7.0; MEGIS software GmbH, Germany). First, visual inspection of the raw recordings was undertaken to detect and interpolate data for bad electrodes. Eyeblink (EOG) and electrocardiogram (EKG) artifacts in the continuous EEG data were manually corrected using a principal component analysis-based adaptive artifact-correction method in BESA. Artifact corrected data for each task were low-pass 
Performance Monitoring ERPs and Triarchic Psychopathy

filtered at $30 \mathrm{~Hz}$ and then segmented into epochs extending from $-400 \mathrm{~ms}$ to $+800 \mathrm{~ms}$ around participant responses in order to capture response-locked ERPs, and from -200 ms to $800 \mathrm{~ms}$ around stimulus presentations in order to extract stimulus-locked ERPs. A semi-automated procedure was then used to detect and reject epochs that contained amplitude deflections of more than $75 \mu \mathrm{V}$ between successive sampling points, or that exceeded an amplitude threshold of $120 \mu \mathrm{V}$, or a low signal threshold of $0.01 \mu \mathrm{V}$, and accepted epochs were converted to the average reference.

\subsection{ERP measurement}

Our main goal in the current study was to examine psychopathy-related variations in ERP measures of error processing, inhibitory control, and flanker interference, as well as to examine patterns of covariance among these ERP measures across the two study tasks (go/no-go, flanker). In the service of this aim, we extracted four ERP measures from each task, two of them error-locked (ERN and Pe) and the other two stimulus-locked (N2, P3). The latter two measures were derived from the no-go condition of the go/no-go task (i.e., no-go N2, no-go P3) and the incongruent condition of the flanker task (incongruent $\mathrm{N} 2$, incongruent $\mathrm{P}$ 3). This analytic approach is consistent with prior studies that have examined covariation among different ERP measures from separate tasks (Nelson et al., 2011; Patrick et al., 2013; Venables et al., 2018), as well as with recent research on error processing in psychopathy (Maurer, Steele, Cope, et al., 2016; Maurer, Steele, Edwards, et al., 2016; Steele et al., 2016) and inhibitory control in externalizing (Brennan \& Baskin-Sommers, 2018), which have typically restricted analyses to absolute ERP amplitude scores for conditions of interest.

Following previous research examining error-related brain activity across tasks, and given that the ERN can begin prior to the completion of the response, we used a -400 $\mathrm{ms}$ to $-200 \mathrm{~ms}$ preresponse interval for baseline correction of the average response-locked 
data (cf. Riesel et al., 2013). Stimulus-locked ERPs were measured on correctly performed trials using a $-200 \mathrm{~ms}$ to $0 \mathrm{~ms}$ prestimulus onset interval for baseline correction.

The selection of electrode sites for quantifying the ERPs of interest was based on the scalp distribution of the current data (see Figure 1), as well as on prior research showing maximal activation at frontocentral electrodes for the ERN and the N200 (Folstein \& van Petten, 2008; Gehring et al., 2012; Larson, Clayson, \& Clawson, 2014) and a centroparietal maximum distribution for the Pe and the P300 (Overbeek et al., 2005; Polich, 2007). Given evidence for superior reliability of ERP measurement across multiple electrodes (cf. Baldwin, Larson, \& Clayson, 2015), we selected a cluster of 20 midline frontocentral electrodes for averaging the ERN's and N200's (HCGSN channel numbers: $5,6,7,8,9,14,15,16,17,21,22,23,24,29,30,186,198,207,215,257$; for electrode configuration, see Figure 1), and a cluster of 20 midline centroparietal electrodes for averaging the Pe's and the P300's from both tasks (HCGSN channel numbers: $45,53,60,79,80,81,88,89,90,100,101,129,130,131$, 132, 142, 143, 144, 155, 257; for electrode configuration, see Figure 1).

Response- and stimulus-locked ERPs were quantified as the mean voltage amplitude within specified time windows, as determined by visual inspection of participants' grand-average waveforms in conjunction with past research. Figure 1 shows the grand-average waveforms and topographical distributions of the different ERP measures examined. ${ }^{1}$ Error trial response-locked ERPs were defined as any response on no-go trials in the go/no-go task (where responses were to be withheld), and as incorrect responses to flanker stimuli in the flanker task. ${ }^{2}$ For the negative-going ERPs quantified at the frontocentral electrode cluster, a 100-ms wide time window was set, spanning from 250 to $350 \mathrm{~ms}$ after stimulus onset for the N2 component (Groom \& Cragg, 2015) and 
Performance Monitoring ERPs and Triarchic Psychopathy

from 0 to 100 ms following responses for the ERN/CRN in both tasks (Riesel et al., 2013). As for positive-going ERPs scored at the centroparietal electrode cluster, the P3 component in the go/no-go task was scored from 350 to $550 \mathrm{~ms}$ following stimulus onset (Groom \& Cragg, 2015), whereas the P3 component in the flanker task was scored between 300 and $550 \mathrm{~ms}$ following stimulus onset, given previous evidence of latency differences between congruent and incongruent trials in this task (e.g., Hajcak et al., 2005). Finally, the more sustained parietal error-positivity (Pe) was measured from 150 to $400 \mathrm{~ms}$ following incorrect responses in each task (Steinhauser \& Yeung, 2012).

\subsection{Data analysis}

Statistical analyses were carried out using the SPSS software package (Version 23, SPSS Inc. Chicago, IL). A winsorization procedure (Wilcox, 2012) was applied to outlying behavioral (RT) and ERP amplitude scores (comprising $2.36 \%$ of all scores) in order to avoid a disproportionate influence of these scores on relations with TriPM scale scores. Specifically, scores higher than the $75^{\text {th }}$ percentile plus 1.5 times the interquartile range of the distribution, or lower than the $25^{\text {th }}$ percentile minus 1.5 the interquartile range of the distribution, were replaced by the maximum or minimum value within these ranges.

Supplemental analyses not directly relevant to our main study hypotheses were carried out for both behavioral and ERP response measures in both tasks and are presented in the Supplementary Materials for completeness. First, reliabilities were computed for each behavioral and ERP measure using split-half (odd versus even trial) correlations, adjusted for attenuation using the Spearman-Brown prophecy formula (cf. Venables et al., 2018). Corrected split-half reliabilities were high in all cases ( $>.82$; see Supplementary Materials), attesting to the internal consistency of measures used to test for hypothesized relationships between psychopathic traits and neural indices of performance monitoring. Second, within-subjects condition effects were tested for both 
behavioral variables in both the go/no-go (go vs. no-go accuracy; correct vs. error trial RTs) and the arrow flanker task (incongruent vs. congruent trial accuracy and RT) to corroborate the validity of the selected task procedures. Third, within-subjects condition effects were also examined to demonstrate a pattern of differential brain reactivity specific to error processing (i.e., error [ERN, Pe] vs. correct [CRN, Pc] response conditions, in both tasks), response inhibition in the go/no-go task (stimulus-locked no-go N2 and P3 vs. go N2 and P3 amplitudes), and flanker interference control in the arrow flanker task (stimulus-locked incongruent N2 and P3 vs. congruent N2 and P3 amplitudes).

Descriptive statistics for the overall sample and for gender subgroups separately, together with independent samples $t$-tests evaluating gender differences in task response measures, are reported in the Supplementary Materials; also reported there are exploratory correlational analyses between TriPM scale scores and the above-mentioned behavioral and ERP variables.

Hierarchical regression analyses were conducted to test our main study hypotheses pertaining to specific links between target ERP response measures (detailed above; see section 2.4) and trait disinhibition. The observed mean-level gender differences in scores for all TriPM scales and some task-response measures (detailed in the Supplementary Materials $)$ were controlled for by including participant gender $(0=$ female; $1=$ male $)$ at Step 1 in these regression analyses. ${ }^{3}$ TriPM Disinhibition scores were included at Step 2 to test for the predictive contribution of this triarchic dimension alone to each task response measure. Finally, TriPM Meanness and TriPM Boldness scores were included at Step 3 to test for any increase in predictive power of these scales for each ERP response measure, as well as to examine the unique contribution of trait disinhibition, after controlling for its overlap with meanness and boldness dimensions at this latter step. Standardized $\beta$ weights and the $\Delta R^{2}$ at each step of the regression models are presented in Table 1 . 
To illustrate the nature of significant $(p<.05)$ effects of TriPM scale scores on ERP measures, figures are presented depicting waveforms for high versus low mediansplit subgroups in the sample as a whole - with men and women grouped as such based on gender-specific medians.

A final set of analyses examined the correlational structure of the ERP measures from the two tasks in order to evaluate the degree to which each indexed common versus distinctive processes across task contexts. Specifically, a principal-axis factor analysis with Promax rotation was performed on the above-noted ERP measures (no-go N2, no-go P3, no-go ERN, no-go Pe, incongruent N2, incongruent P3, flanker ERN, flanker Pe). We used this analytic approach because we sought to (1) characterize associations of the three triarchic dimensions (as indexed by the TriPM) with latent factors reflecting variance shared among different ERP measures (e.g., Nelson et al., 2011; Venables et al., 2018), and (2) evaluate the extent to which each ERP measure operates as an indicator of psychopathic traits as a function of variance in common with, as opposed to distinct from, other ERP measures of interest. Thus, following procedures described by Nelson et al. (2011), we used regression-based estimation to compute factor scores and then used these scores as criterion measures in regression models in which the three TriPM scales where entered as predictors. Then, in a final set of analyses, we examined the extent to which each individual ERP measure - from among those showing significant associations with TriPM scale scores - contained psychopathy-related variance not accounted for by the latent factors reflecting their shared variance, by means of partial correlational analyses.

\section{[INSERT FIGURE 1 ABOUT HERE]}

\section{Results}

Figure 1 presents the grand-average waveforms for all of the ERP measures examined in the current study. 


\subsection{Stimulus-locked ERPs: Response inhibition and flanker interference}

N2: Neither gender nor TriPM scale scores contributed independently to the prediction of no-go N2, $F s<1.57, p \mathrm{~s}>.21, R^{2} s<.022$, or incongruent $\mathrm{N} 2$ amplitudes, $F s$ $<0.54, p \mathrm{~s}>.70, R^{2} s<.015$, at any step of the regression models (see Table 1).

P3: No-go P3 amplitudes were larger for men than for women, as evidenced by a significant positive association with gender at Step $1, F(1,140)=42.49, \beta=.48, p<$ .0001. TriPM Disinhibition scores showed a significant unique predictive contribution to the no-go P3 amplitude at Step $2, \Delta F(1,139)=4.75, \beta=-.16, p=.031$ (See Table 1), with smaller amplitudes, as hypothesized, for high disinhibited participants. TriPM Meanness and Boldness scores did not predict no-go P3 amplitudes at Step 3, $\Delta F(2,137)$ $=0.50, p=.611($ see Table 1$)$, and both gender $(\beta=.52, p<.0001)$ and TriPM Disinhibition scores $(\beta=-.18, p=.040)$ remained significant predictors at this step. Figure 2.A illustrates the effect for TriPM Disinhibition, by depicting no-go P3 waveforms for men and women scoring high versus low (median-split groups) in this scale. The same pattern of results was found for the P3 component derived from the flanker task: men showed larger incongruent $\mathrm{P} 3$ amplitudes than women, $F(1,140)=$ $30.35, \beta=.42, p<.0001$, and TriPM Disinhibition scores uniquely predicted diminished incongruent $\mathrm{P} 3$ amplitudes at Step 2, $\Delta F(1,139)=9.98, \beta=-.24, p=.002$ (see Table 1; Figure 2.C). Inclusion of TriPM Meanness and Boldness scores at Step 3 did not increase explained variance of this ERP measure, $\Delta F(2,137)=1.95, p=.147$, with only gender $(\beta$ $=.48, p<.0001)$ and TriPM Disinhibition scores $(\beta=-.28, p=.001)$ still contributing at this step (see Table 1). ${ }^{4}$

\subsection{Response-Locked ERPs: Error processing}

ERN: ERN amplitude for erroneous (i.e., no-go trial) responses in the go/no-go task did not differ as a function of participant gender, $F(1,140)=0.06, \beta=.02, p=.800$, 
but TriPM Disinhibition scores showed a unique positive association with no-go ERN amplitudes at Step 2, $\Delta F(1,139)=5.39, \beta=.20, p=.022$ (See Table 1), indicative of reduced (i.e., less negative) ERN responding among participants scoring higher in disinhibition. Predictive contributions to no-go ERN amplitudes for the other two TriPM scales scores (Meanness, Boldness) at Step 3 were negligible, $\Delta F(2,137)=0.50, p=.611$ (See Table 1), with only TriPM Disinhibition scores showing again a significant unique positive association $(\beta=.20, p=.040)$ with this response measure at this step. Figure 2.E illustrates the result for TriPM Disinhibition, depicting reduced (i.e., less negative) no-go ERN amplitude for participants scoring high as compared to low in trait disinhibition. By contrast, neither gender nor TriPM scale scores evidenced any significant predictive contribution to the ERN response in the arrow flanker task, $F s<0.18, p \mathrm{~s}>.83, R^{2} s<$ .003 , at any step of the regression model (See Table 1$)^{5}$.

Of note, and in line with prior research (e.g., Burwell et al., 2016; Riesel et al., 2013), the correlation between ERNs from the two tasks in the current study was moderate, $r=.45, p<.0001$ (see Table 2). To further explore the differential relations found for these two variants of ERN in relation to the trait of disinhibition, we conducted a multiple regression analysis in which both no-go ERN and flanker ERN amplitude scores were entered concurrently as predictors of TriPM Disinhibition. The overall model was significant, $F(2,139)=4.49, p=.013, R^{2}=0.06$, with only the no-go ERN contributing significantly to prediction of TriPM Disinhibition scores - in a positive direction $(\beta=.27, p=.004)$, indicating a reduced no-go ERN amplitude for individuals scoring higher in disinhibition. Interestingly, the unique variance in flanker ERN amplitude (i.e., that unrelated to no-go ERN) showed an opposite directional effect, albeit nonsignificant $(\beta=-.17, p=.065)$. To facilitate comparison with the findings for the no- 
go ERN, Figure 2.G shows grand-average flanker ERN waveforms for participants scoring above (high) versus below the median (low) on TriPM Disinhibition.

Pe: Pe amplitude following erroneous "go" responses in the go/no-go task was larger for men than for women, $F(1,140)=18.99, \beta=.35, p<.0001$. At Step 2, TriPM Disinhibition scores showed a significant predictive contribution to no-go Pe amplitudes, $\Delta F(1,139)=4.68, \beta=-.17, p=.032$, indicative of reduced amplitudes of this ERP response for participants scoring high in disinhibition (see Table 1 and Figure 2.I). Neither TriPM Meanness nor TriPM Boldness scores contributed to the prediction of nogo Pe amplitudes at Step 3, $\Delta F(2,137)=1.17, p=.314($ see Table 1$)$, on which both gender $(\beta=.40, p<.0001)$ and TriPM Disinhibition scores $(\beta=-.19, p=.037)$ remained as significant predictors. For the flanker task, men showed larger mean Pe amplitudes than women, $F(1,140)=21.62, \beta=.37, p<.0001$, and TriPM Disinhibition scores evidenced a significant predictive contribution to flanker Pe amplitudes at Step 2, $\Delta F(1$, $139)=6.97, \beta=-.21, p=.009$, with amplitudes again smaller for participants scoring high in disinhibition (see Figure 2.K). No unique predictive contribution was evident for the other two TriPM scale scores when included at Step 3, $\Delta F(2,137)=2.00, p=.139$ (see Table 1$)$, with only gender $(\beta=.42, p<.0001)$ and TriPM Disinhibition $(\beta=-.24, p=$ $.008)$ remaining as significant predictors. ${ }^{6}$

\section{[INSERT FIGURE 2/TABLE 1 ABOUT HERE]}

\subsection{Covariance among ERP measures and triarchic dimensions}

As can be seen in the results from correlational analyses presented in Table 2, there was a good deal of overlap among the various ERP measures examined. The strongest overlap between counterpart ERP measures from the two tasks was found for the Pe $(r=.68)$, followed by the P3 $(r=.61)$, the ERN $(r=.45)$, and the N2 $(r=.39)$. Across the two tasks, P3 and Pe measures were highly inter-correlated (mean $r=0.69$; range $=$ 
Performance Monitoring ERPs and Triarchic Psychopathy

$.61-.83$ ), and the ERN and N2 measures showed moderate-level overlap (mean $r=0.48$; range $=.24-.71)$. The overlap between frontal negativity $(\mathrm{N} 2$ and $\mathrm{ERN})$ and parietal positivity $(\mathrm{P} 3$ and $\mathrm{Pe})$ measures was lower (mean $r=.18$; range $=.05-.39)$ than that between N2/ERN measures or P3/Pe measures.

Results from a principal-axis factor analysis with Promax rotation (Bartlett's $\chi^{2}=$ $654.84, d f=28, p<.0001 ; \mathrm{KMO}=.708)$ revealed that the correlations among the various ERP measures could be represented by two factors (eigenvalues $=3.60,1.98$; all subsequent values $\leq .92$ ), which accounted for $69.73 \%$ of the total variance in scores. P3 and Pe measures loaded uniformly onto the first factor (labeled the 'P3/Pe' factor; range of loadings $=.80-.87$ ), which explained $45.04 \%$ of the total score variance. The second factor was defined by N2 and ERN measures ('N2/ERN' factor; loading range $=.63-$ .85 ), and explained $24.69 \%$ of variance. Loadings for the eight different ERP measures on the two extracted factors are shown in Table 2 (right side).

\section{[INSERT TABLE 2 ABOUT HERE]}

Factor scores were computed via the regression method and evaluated for associations with TriPM scale scores using the same hierarchical regression analytic approach described above for each individual ERP measure. Results from this set of analyses are reported in Table 3. For P3/Pe factor scores, gender emerged as a significant predictor at Step1, $F(1,140)=31.56, \beta=.43, p<.0001$, and TriPM Disinhibition evidenced a significant negative association at Step $2, \Delta F(1,139)=8.51, \beta=-.22, p=$ .004. TriPM Meannes and Boldness scores did not account for additional variance in $\mathrm{P} 3 / \mathrm{Pe}$ factor scores at Step 3, $\Delta F(2,137)=1.78, p=.173$ (see Table 3), and significant prediction was retained for both gender $(\beta=.49, p<.0001 ; \mathrm{P} 3 / \mathrm{Pe}$ factor scores greater for men than women) and TriPM Disinhibition ( $\beta=-.25, p=.004$; P3/Pe factor scores lower for participants scoring higher in disinhibition). By contrast, neither gender nor TriPM 
scale scores showed unique predictive contributions for N2/ERN factor scores, $F s<0.37$, $p \mathrm{~s}>.54, R^{2} s<.003$, at any step (see Table 3 ).

A final set of analyses examined the extent to which variance shared among the different ERP indicators, reflected in P3/Pe and N2/ERN factor scores, could account for observed associations of particular ERP measures with TriPM Disinhibition (i.e., P3 and Pe from each task, no-go ERN from the go/no-go task) when controlling for participant gender. Partial correlations were computed for each P3 and Pe measure with TriPM Disinhibition, controlling for P3/Pe factor scores and gender. These analyses did not reveal a unique predictive relationship for any individual $\mathrm{P} 3$ or Pe measure with TriPM Disinhibition scores (partial $r=.01, p=.908$, for no-go P3; partial $r=.09, p=.278$, for no-go Pe; partial $r=-.11, p=.209$ for incongruent $\mathrm{P} 3$; partial $r=-.04, p=.649$ for flanker Pe). The implication is that the shared variance among the P3 and Pe measures reflects some process in common, related to disinhibition, that accounts for their individual associations with this triarchic trait dimension.

The same analysis was performed for the no-go ERN measure, which was found to be significantly associated with TriPM Disinhibition scores (see Table 1), and showed substantial overlap with the other N2 and ERN measures studied (see Table 2). In this case, the relationship between the no-go ERN and TriPM Disinhibition scores remained significant when controlling for N2/ERN factor scores and gender (partial $r=.24, p=$ .004), indicating that the no-go ERN contained distinct disinhibition-related variance that was not shared with the other ERN and N2 measures studied.

[INSERT TABLE 3 ABOUT HERE]

\section{Discussion}

The broad aim of the current study was to test for associations of electrophysiological indices of interference control, response inhibition, and error 
Performance Monitoring ERPs and Triarchic Psychopathy

monitoring, derived from two different performance tasks (go/no-go and flanker), with dimensions of psychopathy as described by the triarchic model of psychopathy (Patrick et al., 2009). We found selective associations between the disinhibition dimension and certain ERP indices of performance monitoring previously linked to externalizing problems and traits, along lines consistent with dual-process models of psychopathy (Fowles \& Dindo, 2006, 2009; Patrick \& Bernat, 2009), which posit that deficits in cognitive control processes underlie the impulsive-antisocial features of this personality disorder that relate most to externalizing proneness. The current study also found evidence of commonalities among the different ERP indices of performance monitoring across tasks, allowing for inferences about specific neurocognitive processes linked to this dimension of psychopathy.

\subsection{Action selection: Response inhibition and flanker interference}

With regard to ERP indicators of response inhibition and flanker interference control, we did not find any significant associations for disinhibition or other triarchic dimensions with $\mathrm{N} 2$ amplitude in either the go/no-go or the flanker task. Considering that N2 has been linked to monitoring of response conflict in tasks of these types (for reviews, see Huster et al., 2013; Larson et al., 2014), our results do not provide evidence of psychopathy-related deviations in neural processing of preresponse conflict during action selection in interference (e.g., flanker) or response inhibition (e.g., go/no-go) tasks, at least in this undergraduate test sample.

A contrasting pattern of results emerged for stimulus P3 amplitude derived from the two tasks, which was associated negatively in each case with the triarchic dimension of disinhibition. This accords with prior research evidence for reduced no-go P3 amplitude in participants scoring high in externalizing proneness (Brennan \& BaskinSommers, 2018) or self-reported psychopathic and motoric-impulsiveness traits (Kim \& 
Jung, 2014), as well as with research demonstrating blunted flanker-P3 amplitude in relation to higher reported externalizing tendencies (Nelson et al., 2011; Venables et al., 2018). Collectively, the observed disinhibition-P3 associations provide strong support for dual-process models of psychopathy, which posit that brain response indicators of externalizing proneness - such as reduced amplitude of the stimulus-elicited P3 (Gao \& Raine, 2009; Pasion et al., 2018) - should be selectively related to the impulsive and antisocial features of psychopathy (Fowles \& Dindo, 2006, 2009; Patrick \& Bernat, 2009). Hence, our results dovetail with previous reports showing reduced amplitude of visual-oddball $\mathrm{P} 3$ response in relation to higher levels of the impulsive-antisocial features of psychopathy in both incarcerated and nonincarcerated samples (Carlson, Thái, \& McLarnon, 2009; Venables \& Patrick, 2014), and indicate that this relationship may generalize across different tasks, as suggested by other published work (e.g., Bernat et al., 2011; Nelson et al., 2011; Patrick et al., 2013; Perkins et al., 2017; Venables et al., 2018).

\subsection{Error processing}

Regarding the ERN, theorized to index detection and processing of errors, our results demonstrated reduced amplitude in relation to triarchic disinhibition for the variant of this response derived from the go/no-go task, but not the flanker task. These results partially corroborate previous findings showing reduced amplitude of ERN responding in nonincarcerated participants scoring high in externalizing tendencies (Hall et al., 2007) or impulsive-antisocial features of psychopathy (Bresin et al., 2014; Heritage \& Benning, 2013; Pasion et al., 2016), and are consistent with a recent report showing the latter variant of ERN (i.e., from the arrow flanker task) to be unrelated to scale measures of trait disinhibition (Venables et al., 2018). In this regard, our results indicate that the relationship between trait disinhibition and the ERN may be contingent upon the specific experimental paradigm in which it is measured, or the type of error in relation to which it 
Performance Monitoring ERPs and Triarchic Psychopathy

is assessed. In line with previous studies, we found a moderate correlation between counterpart ERNs from the go/no-go and flanker tasks ( $r=.45$; see Burwell et al., 2016; Riesel et al., 2013), suggesting the presence of both common and task-specific influences on error-related brain activity across the two tasks.

Interestingly, regression analyses in which both no-go ERN and flanker ERN were entered concurrently as predictors of disinhibition scores revealed that the unique portions of variance in each were related in opposing directions to disinhibition, with the no-go ERN predicting significantly and positively - indicative of diminished no-go ERN amplitude for high- versus-low disinhibited participants - and the flanker ERN showing a nonsignificant relationship in the opposing direction. Thus, our results indicate that it was only the unique variance in no-go ERN amplitude, rather than the variance it shared with flanker ERN amplitude, that related inversely to disinhibition scores as reported in prior published studies of nonincarcerated individuals assessed for psychopathy (Bresin et al., 2014; Heritage \& Benning, 2013; Pasion et al., 2016). Collectively, our results provide the first empirical support for the conclusion advanced in a recent meta-analytic paper (cf. Pasion \& Barbosa, 2019) that no-go variants of the ERN response exhibit stronger associations with externalizing proneness than ERN variants assessed in other response conflict tasks.

Our findings for these ERN variants highlight the importance of considering the contribution of task parameters or context in the study of individual differences in error processing (Weinberg et al., 2015). Reduced amplitude of the ERN in high externalizing/ disinhibited populations has been proposed as a mechanism to explain their tendency to continually repeat maladaptive or harmful behaviors (Hall et al., 2007), perhaps linked to a reduced ability to self-monitor errors or reflect upon actions following mistakes (Falkenstein et al., 2000; Gehring et al., 1993; Holroyd \& Coles, 2002; Yeung et al., 
2004). Whatever specific neurobiological process might best account for this effect, our results suggest that reduced ERN is most clearly observed when high disinhibited participants fail to inhibit prepotent response tendencies (i.e., following response inhibition errors), rather than after action slips due to interference.

As for later stages of error processing, negative associations with triarchic disinhibition were found for both no-go and flanker task variants of the Pe. Though prior studies with nonincarcerated samples assessed for psychopathy did not report analyses for this error-locked ERP component (Bresin et al., 2014; Heritage \& Benning, 2013; Pasion et al., 2016), and studies with incarcerated samples have yielded inconsistent findings for this measure (e.g., Maurer, Steele, Edwards et al., 2016; Maurer, Steele, Cope et al., 2016; Steele et al., 2016), our results are consistent with a recent study showing reduced Pe amplitude in an arrow flanker task in participants scoring high on self-report measures of trait disinhibition (Venables et al., 2018). As discussed further below, the convergence found among variants of P3 and Pe from the two tasks in the current study - and in their relationships with disinhibition - suggests that the observed Pe reductions among high disinhibited participants reflect some process in common with the P3 that arises at later stages of error processing (Overbeek et al., 2005; see also Venables et al., 2018).

\subsection{Covariance among performance monitoring ERPs}

In addition to testing for associations of ERP indicators of response inhibition, flanker interference, and error monitoring from each task with triarchic psychopathy dimensions, we also examined patterns of covariance among the different performance monitoring-related ERPs from these two tasks. Substantial overlap was in fact observed among the different ERPs across tasks and processing contexts, with a factor analysis revealing two distinct factors accounting for their covariance - one, labeled ' $\mathrm{P} 3 / \mathrm{Pe}$ ', reflecting variance in common among the $\mathrm{P} 3$ and Pe measures studied, and the other, 
labeled 'N2/ERN', reflecting variance shared among measures of N2 and ERN from the two tasks. The P3/Pe factor appeared highly similar to one recently reported by Burwell et al. (2016), and together with this and other work showing high correlations among P3 and Pe measures (Cassidy et al., 2012; Davies et al., 2001), our results suggest that both these components are indicative of neurocognitive processes involved in later, elaborative stages in the processing of motivationally significant events, such as imperative target stimuli or errors in performance (e.g., Burwell et al., 2016; Davies et al., 2001; Overbeek et al., 2005; Ridderinkhof et al., 2009). On the other hand, the coherence observed among ERN and N2 measures from the two tasks appears consistent with work showing that both stimulus- and response-locked frontal negativities measured in performance-monitoring tasks are generated in partially overlapping areas of the medial frontal cortex (MFC), including the anterior cingulate cortex (ACC) and the presupplementary motor area (e.g., Amodio et al., 2008; Dehaene, Posner, \& Tucker, 1994; Gruendler et al., 2011; Nieuwenhuis et al., 2003; van Veen \& Carter, 2002). Additionally, these ERP components are each characterized by prominent theta-frequency EEG activity (e.g., Cavanagh, Zambrano-Vazquez, \& Allen, 2012; Nigbur, Ivanova, \& Stürmer, 2011), perhaps reflecting a common process of attentional control following events that signal the potential need for alterations in behavior (Van Noordt, Campopiano, \& Segalowitz, 2016). Relatedly, the conflict monitoring theory (Yeung et al., 2004; see Larson et al., 2014 for a review) posits that the ERN and the N2 represent indicators of ACC-mediated postresponse and preresponse conflict processing, respectively.

Despite this observed covariation, N2/ERN factor scores were unrelated to the triarchic dimensions, and the no-go ERN showed a predictive association with disinhibition scores separate from the variance it shared with the other N2 and ERN measures studied. This finding, together with other work reporting null relationships 
between externalizing proneness (disinhibition) and other ERPs believed to share similarities with the ERN - such as the feedback-related negativity (Bernat et al., 2011; see also Schulreich, Pfabigan, Derntl, \& Sailer, 2013) - suggests that high trait disinhibition is not characterized by global impairments in early stages of (putatively) ACC-mediated control functions across different tasks and tasks contexts. Rather, such reduced neural activity appears more circumscribed to task contexts that engage inhibitory mechanisms, and more specifically, to conditions calling for inhibition of prepotent response tendencies - such as the no-go condition of the go/no-go task.

A contrasting pattern of results was evident for $\mathrm{P} 3 / \mathrm{Pe}$ factor scores, which were inversely related to disinhibition scores and accounted for the associations of each P3 and Pe measure with this triarchic dimension. In this regard, our results dovetail with previous work demonstrating convergence of scale measures of disinhibition with distinct variants of P3 response (e.g., Patrick et al., 2013), including Pe from the flanker-task (cf. Venables et al., 2018). Collectively, our results provide support for the theoretical perspective that the processing style of highly disinhibited individuals entails deviations in elaborativeassociative processing of stimulus events and response outcomes, and its integration with memory representations of task goals, a process that may be essential to anticipation, reflection, and self-regulation (cf. Patrick, 2018; see also Perkins et al., 2017).

\subsection{Limitations and future directions}

The present study has some limitations that may limit the generalizability of our findings and that highlight directions for future research. First, our study focused on an undergraduate sample with enhanced $(\sim 70 \%)$ representation of women, unselected for psychopathy scores. Further studies with larger community samples preselected for varying levels of psychopathic traits and exhibiting greater heterogeneity with respect to age, educational level, and economic status are needed to establish the generalizability of 
Performance Monitoring ERPs and Triarchic Psychopathy

our findings. Also, the overrepresentation of women relative to men in our sample posed somewhat of a limitation in terms of testing for moderating effects of gender on psychopathy/ERP associations. This was particularly important given that significant gender differences were observed in mean levels of both TriPM scale scores and certain behavioral and ERP measures - in particular, P3 and Pe (see Supplementary materials). However, with gender controlled for in our analyses, a coherent pattern of associations was observed for the P3 and Pe in relation to TriPM Disinhibition scores, and regression models incorporating interaction terms for gender revealed no such effects, indicating that observed relations between disinhibition scores and ERP performance-monitoring measures did not vary as a function of gender.

Moreover, while research on the neural correlates of psychopathic traits in nonoffender samples is vital to an etiological understanding of the full continuum of the psychopathy construct (Patrick, 2018), and variations in its expression (Skeem et al., 2011), our results need to be replicated and extended to other populations with more severe manifestations of psychopathic personality, such as incarcerated offenders. This is particularly important given that patterns of results from studies of incarcerated offenders, for go/no-go tasks in particular, have been mixed and are not easily integrated with current study findings (see Maurer, Steele, Cope et al., 2016; Maurer, Steele, Edwards et al., 2016; Steele et al., 2016). One probable source of inconsistent findings across studies of this type is differences in methods used to assess psychopathic traits. As discussed elsewhere (Pasion et al., 2018; Schulreich, 2016), measures such as the TriPM, employed in the current study, are designed to operationalize psychopathy in terms of traits that map onto neurobehavioral dimensions such as trait fearlessness and externalizing proneness emphasized in dual-process models of psychopathy (Patrick \& Bernat, 2009; see also Skeem et al., 2011). Clinically based measures such as the PCL-R and inventories derived 
from or patterned after it assess psychopathy in terms of correlated symptom dimensions not referenced directly to neurobehavioral constructs or measures. In future studies with incarcerated populations, it will be valuable to collect measures of the triarchic trait dimensions - such as the TriPM, or the many alternative scale sets that have been developed to assess these trait dimensions (Patrick \& Drislane, 2015; see also, e.g., Drislane, Brislin, Jones, \& Patrick, 2018; Ruchensky, Donnellan, \& Edens, 2018) - as a complement to clinical symptom measures such as the PCL-R. Doing so would allow for tests of possible differences in relations for alternative measures of psychopathic dimensions with ERP indicators of performance monitoring (see Schulreich, 2016, for a similar argument), and evaluation of the generalizability of current findings to clinical participants.

In addition, to further advance our understanding of distinct neurocognitive processes underlying performance monitoring and the role they play in behavior disorders, it would be useful in future studies to employ hybrid paradigms that allow for assessment of processes related to different types of errors, and/or to inhibitory vs. interference control processing, within the same task (see, for example, Vilà-Balló et al., 2014). Furthermore, it should be noted that the observed relations between psychopathic traits and performance-monitoring ERPs in the current study were generally not accompanied by significant differences in accuracy or reaction time (see Supplementary Materials). Although it is common for studies documenting externalizing- or disinhibition-related impairments in performance-related ERP response to report null effects for behavioral measures (e.g., Hall et al., 2007; Kim \& Jung, 2014; Pasion et al., 2016; but see Heritage \& Benning, 2013), future studies should seek to clarify the basis of this dissociation in effects - for example, by assessing a broader range of behavioral 
Performance Monitoring ERPs and Triarchic Psychopathy

indicators of distinct executive control functions, including ones known to exhibit associations with disinhibition (e.g., Venables et al., 2018; Young et al., 2009).

Finally, we wish to note that our factor analytic solution should not be interpreted as indicating full equivalence of the neural processes indexed by N2 and ERN measures, or by $\mathrm{P} 3$ and Pe measures. Rather, our approach should be viewed as a promising avenue to better characterize the overlap vs. distinctiveness of neural measures of interest in relation to individual difference trait constructs (e.g., Patrick \& Hajcak, 2016). The high degree of overlap observed among these measures across tasks and task contexts could also reflect partial overlap in task designs, as our two study tasks constitute speeded response conflict tasks that may assess different, albeit partially correlated, processes. Furthermore, it is also likely that different factor solutions might arise for ERPs other than the ones examined in our study (see Burwell et al., 2016).

Notwithstanding these limitations, our study represents an important contribution to characterizing deviations in neural indicators of performance monitoring in relation to psychopathic traits. On one hand, our results yield support for dual-process models of psychopathy (Fowles \& Dindo, 2006, 2009; Patrick \& Bernat, 2009) by showing that ERP indicators of externalizing proneness - such as reduced amplitudes of the ERN and P3 components (Patrick \& Bernat, 2009) - are associated selectively with impulsiveantisocial features of psychopathy represented in the triarchic dimension of disinhibition (Patrick \& Drislane, 2015; Patrick et al., 2009). In addition, our findings provide further insight into the processing style of high-disinhibited participants within performance monitoring tasks, in at least two important ways. First, our results suggest that disinhibition is mainly associated with reduced amplitudes of ERP components implicated in later, more elaborative stages in the processing of motivationally significant events across different tasks and task contexts. Second, disinhibition-related impairment in ERPs 
Performance Monitoring ERPs and Triarchic Psychopathy

indexing early, more automatic processing of stimulus features and enacted responses appeared circumscribed to conditions involving failures in inhibiting prepotent response tendencies - as evidenced by reduced no-go ERN within the go/no-go task, but not the flanker task. In this regard, our results highlight the importance of further investigating variations in the psychometric properties of, and degree of convergence among, different performance monitoring ERPs across tasks (e.g., Patrick \& Hajcak, 2016). Work of this kind is likely to have important implications for advancing our understanding of psychopathy - and of other clinical problems more broadly - in neurobehavioral terms. 
Performance Monitoring ERPs and Triarchic Psychopathy

\section{References}

Albert, J., López-Martín, S., Hinojosa, J. A., \& Carretié, L. (2013). Spatiotemporal characterization of response inhibition. Neuroimage, 76, 272-281. https://doi.org/10.1016/j.neuroimage.2013.03.011

Amodio, D. M., Master, S. L., Yee, C. M., \& Taylor, S. E. (2008). Neurocognitive components of the behavioral inhibition and activation systems: Implications for theories of self-regulation. Psychophysiology, 45(1), 11-19. https://doi.org/10.1111/j.1469-8986.2007.00609.x

Arbel, Y., \& Donchin, E. (2009). Parsing the componential structure of post-error ERPs: A principal component analysis of ERPs following errors. Psychophysiology, 46(6), 1179-1189. https://doi.org/10.1111/j.1469-8986.2009.00857.x

Baldwin, S. A., Larson, M. J., \& Clayson, P. E. (2015). The dependability of electrophysiological measurements of performance monitoring in a clinical sample: A generalizability and decision analysis of the ERN and Pe. Psychophysiology, 52(6), 790-800. https://doi.org/10.1111/psyp.12401

Bernat, E. M., Nelson, L. D., Steele, V. R., Gehring, W. J., \& Patrick, C. J. (2011). Externalizing psychopathology and gain-loss feedback in a simulated gambling task: Dissociable components of brain response revealed by time-frequency analysis. Journal of Abnormal Psychology, 120(2), 352-364. https://doi.org/10.1037/a0022124

Brazil, I. A., de Bruijn, E., Bulten, B., von Borries, A., van Lankveld, J., Buitelaar,J., \& Verkes, R. J. (2009). Early and late components of error monitoring in violent offenders with psychopathy. Biological Psychiatry, 65(2), 137-143. https://doi.org/10.1016/j.biopsych.2008.08.011

Brennan, G. M., \& Baskin-Sommers, A. R. (2018). Brain-behavior relationships in externalizing: P3 amplitude reduction reflects deficient inhibitory 
Performance Monitoring ERPs and Triarchic Psychopathy

control. Behavioural Brain Research, 337, 70-79.

https://doi.org/10.1016/j.bbr.2017.09.045

Bresin, K., Finy, M. S., Sprague, J., \& Verona, E. (2014). Response monitoring and adjustment: Differential relations with psychopathic traits. Journal of Abnormal Psychology, 123(3), 634-649. https://doi.org/10.1037/a0037229

Burwell, S. J., Malone, S. M., \& Iacono, W. G. (2016). One-year developmental stability and covariance among oddball, novelty, go/no-go, and flanker event-related potentials in adolescence: A monozygotic twin study. Psychophysiology, 53, 991-1007. https://doi.org/10.1111/psyp.12646

Carlson, S. R., Thái, S., \& McLarnon, M. E. (2009). Visual P3 amplitude and self-reported psychopathic personality traits: Frontal reduction is associated with self-centered impulsivity. Psychophysiology, 46(1), 100-113. https://doi.org/10.1111/j.1469$\underline{8986.2008 .00756 . x}$

Cassidy, S. M., Robertson, I. H., \& O'Connell, R. G. (2012). Retest reliability of event-related potentials: Evidence from a variety of paradigms. Psychophysiology, 49(5), 659-664. https://doi.org/10.1111/j.1469$\underline{8986.2011 .01349 . x}$

Cavanagh, J. F., Zambrano-Vazquez, L., \& Allen, J. J. (2012). Theta lingua franca: A common mid-frontal substrate for action monitoring processes. Psychophysiology, 49(2), 220-238. https://doi.org/10.1111/j.1469$\underline{8986.2011 .01293 . \mathrm{x}}$

Cleckley, H. (1941/1976). The mask of sanity. St. Louis, MO: Mosby.

Coles, M. G., Scheffers, M. K., \& Holroyd, C. B. (2001). Why is there an ERN/Ne on correct trials? Response representations, stimulus-related components, and the theory of error-processing. Biological Psychology, 56(3), 173-189. https://doi.org/10.1016/S0301-0511(01)00076-X 
Performance Monitoring ERPs and Triarchic Psychopathy

Davies, P. L., Segalowitz, S. J., Dywan, J., \& Pailing, P. E. (2001). Error-negativity and positivity as they relate to other ERP indices of attentional control and stimulus processing. Biological Psychology, 56(3), 191-206. https://doi.org/10.1016/S0301$\underline{0511(01) 00080-1}$

Dehaene, S., Posner, M. I., \& Tucker, D. M. (1994). Localization of a neural system for error detection and compensation. Psychological Science, 5(5), 303-305. https://doi.org/10.1111/j.1467-9280.1994.tb00630.x

Donchin, E., \& Coles, M. G. (1988). Is the P300 component a manifestation of context updating?. Behavioral and Brain Sciences, 11(3), 357-374. https://doi.org/10.1017/S0140525X00058027

Drislane, L. E., Brislin, S. J., Jones, S., \& Patrick, C. J. (2017). Interfacing five-factor model and triarchic conceptualizations of psychopathy. Psychological Assessment, 30(6), 834-840. https://doi.org/10.1037/pas0000544

Eriksen, B. A., \& Eriksen, C. W. (1974). Effects of noise letters upon the identification of a target letter in a non-search task. Perception \& Psychophysics, 16, 143-149. https://doi.org/10.3758/BF03203267

Esteller, À., Poy, R., \& Moltó, J. (2016). Deficient aversive-potentiated startle and the Triarchic model of psychopathy: The role of boldness. Biological Psychology, 117, 131-140. https://doi.org/10.1016/j.biopsycho.2016.03.012

Euser, A. S., Arends, L. R., Evans, B. E., Greaves-Lord, K., Huizink, A. C., \& Franken, I. H. (2012). The P300 event-related brain potential as a neurobiological endophenotype for substance use disorders: a meta-analytic investigation. Neuroscience \& Biobehavioral Reviews, 36(1), 572-603. https://doi.org/10.1016/j.neubiorev.2011.09.002

Falkenstein, M., Hohnsbein, J., Hoormann, J., \& Blanke, L. (1991). Effects of crossmodal divided attention on late ERP components. II. Error processing in choice reaction 
Performance Monitoring ERPs and Triarchic Psychopathy

tasks. Electroencephalography and Clinical Neurophysiology, 78(6), 447-455. https://doi.org/10.1016/0013-4694(91)90062-9

Falkenstein, M., Hoormann, J., Christ, S., \& Hohnsbein, J. (2000). ERP components on reaction errors and their functional significance: a tutorial. Biological Psychology, 51(2-3), 87-107. https://doi.org/10.1016/S0301-0511(99)00031-9

Falkenstein, M., Hoormann, J., \& Hohnsbein, J. (1999). ERP components in Go/Nogo tasks and their relation to inhibition. Acta Psychologica, 101, 267-291. https://doi.org/10.1016/S0001-6918(99)00008-6

Fischer, A. G., Klein, T. A., \& Ullsperger, M. (2017). Comparing the error-related negativity across groups: The impact of error- and trial-number differences. Psychophysiology, 54(7), 998 1009. https://doi.org/10.1111/psyp.12863

Folstein, J. R., \& Van Petten, C. (2008). Influence of cognitive control and mismatch on the N2 component of the ERP: a review. Psychophysiology, 45(1), 152-170. https://doi.org/10.1111/j.1469-8986.2007.00602.x

Fowles, D.C., \& Dindo, L. (2006) A dual-deficit model of psychopathy. In C. J. Patrick (Ed.), Handbook of psychopathy (pp. 14-34). New York: Guilford Press.

Fowles, D. C., \& Dindo, L. (2009). Temperament and psychopathy: A dual-pathway model. Current Directions in Psychological Science, 18, 179-183. https://doi.org/10.1111/j.1467-8721.2009.01632.x

Gao, Y., \& Raine, A. (2009). P3 event-related potential impairments in antisocial and psychopathic individuals: A meta-analysis. Biological Psychology, 82, 199-210. https://doi.org/10.1016/j.biopsycho.2009.06.006

Gehring, W. J., Goss, B., Coles, M. G., Meyer, D. E., \& Donchin, E. (1993). A neural system for error detection and compensation. Psychological Science, 4(6), 385-390. https://doi.org/10.1111/j.1467-9280.1993.tb00586.x 
Performance Monitoring ERPs and Triarchic Psychopathy

Gehring, W.J., Liu, Y., Orr, J.M., \& Carp, J. (2012). The error-related negativity (ERN/Ne). In S. J. Luck and E. S. Kappenman (Eds.), The Oxford Handbook of Event-related Potential Components, 231-300. New York: Oxford University Press. https://doi.org/10.1093/oxfordhb/9780195374148.013.0120

Groom, M. J., \& Cragg, L. (2015). Differential modulation of the N2 and P3 eventrelated potentials by response conflict and inhibition. Brain and cognition, 97, 19. https://doi.org/10.1016/j.bandc.2015.04.004

Gruendler, T. O., Ullsperger, M., \& Huster, R. J. (2011). Event-related potential correlates of performance-monitoring in a lateralized time-estimation task. PloS one, 6(10), e25591. https://doi.org/10.1371/journal.pone.0025591

Hajcak, G., Meyer, A., \& Kotov, R. (2017). Psychometrics and the neuroscience of individual differences: Internal consistency limits between-subjects effects. Journal of abnormal psychology, 126(6), 823-834. https://doi.org/10.1037/abn0000274

Hajcak, G., Moser, J. S., Yeung, N., \& Simons, R. F. (2005). On the ERN and the significance of errors. Psychophysiology, 42(2), 151-160. https://doi.org/10.1111/j.1469-8986.2005.00270.x

Hall, J. R., Bernat, E. M., \& Patrick, C. J. (2007). Externalizing psychopathology and the error-related negativity. Psychological Science, 18, 326-333. https://doi.org/10.1111/j.1467-9280.2007.01899.x

Hare, R.D. (2003). The Hare Psychopathy Checklist-Revised (2nd ed.). Toronto, ON: MHS.

Heritage, A. J., \& Benning, S. D. (2013). Impulsivity and response modulation deficits in psychopathy: Evidence from the ERN and N1. Journal of Abnormal Psychology, 122(1), 215-222. https://doi.org/10.1037/a0030039

Holroyd, C. B., \& Coles, M. G. (2002). The neural basis of human error processing: Reinforcement learning, dopamine, and the error-related negativity. Psychological Review, 109(4), 679-709. https://doi.org/10.1037/0033-295X.109.4.679 
Performance Monitoring ERPs and Triarchic Psychopathy

Huster, R. J., Enriquez-Geppert, S., Lavallee, C. F., Falkenstein, M., \& Herrmann, C. S. (2013). Electroencephalography of response inhibition tasks: Functional networks and cognitive contributions. International Journal of Psychophysiology, 87(3), 217233. https://doi.org/10.1016/j.ijpsycho.2012.08.001

Iacono, W. G., Malone, S. M., \& McGue, M. (2003). Substance use disorders, externalizing psychopathology, and P300 event-related potential amplitude. International Journal of Psychophysiology, 48(2), 147-178. https://doi.org/10.1016/S0167-8760(03)00052$\underline{7}$

Jodo, E., \& Kayama, Y. (1992). Relation of a negative ERP component to response inhibition in a Go/No-go task. Electroencephalography and Clinical Neurophysiology, 82(6), 477-482. https://doi.org/10.1016/0013-4694(92)90054-L

Kiehl, K. A., Smith, A. M., Hare, R. D., \& Liddle, P. F. (2000). An event-related potential investigation of response inhibition in schizophrenia and psychopathy. Biological Psychiatry, 48(3), 210-221. https://doi.org/10.1016/S0006-3223(00)00834-9

Kim, Y. Y., \& Jung, Y. S. (2014). Reduced frontal activity during response inhibition in individuals with psychopathic traits: An sLORETA study. Biological Psychology, 97, 49-59. https://doi.org/10.1016/j.biopsycho.2014.02.004

Kok, A. (2001). On the utility of P3 amplitude as a measure of processing capacity. Psychophysiology, 38(3), 557-577. https://doi.org/10.1017/S0048577201990559

Kopp, B., Mattler, U., Goertz, R., \& Rist, F. (1996). N2, P3 and the lateralized readiness potential in a nogo task involving selective response priming. Electroencephalography and Clinical Neurophysiology, 99(1), 19-27. https://doi.org/10.1016/0921-884X(96)95617-9

Kopp, B., Rist, F., \& Mattler, U. W. E. (1996). N200 in the flanker task as a neurobehavioral tool for investigating executive control. Psychophysiology, 33(3), 282-294. https://doi.org/10.1111/j.1469-8986.1996.tb00425.x 
Performance Monitoring ERPs and Triarchic Psychopathy

Krueger, R. F., Hicks, B. M., Patrick, C. J., Carlson, S. R., Iacono, W. G., \& McGue, M. (2002). Etiologic connections among substance dependence, antisocial behavior and personality: Modeling the externalizing spectrum. Journal of Abnormal Psychology, 111(3), 411-424. https://doi.org/10.1037/0021-843X.111.3.411

Krueger, R. F., Markon, K. E., Patrick, C. J., Benning, S. D., \& Kramer, M. D. (2007). Linking antisocial behavior, substance use, and personality: an integrative quantitative model of the adult externalizing spectrum. Journal of Abnormal Psychology, 116(4), 645-666. https://doi.org/10.1037/0021-843X.116.4.645

Larson, M. J., Clayson, P. E., \& Clawson, A. (2014). Making sense of all the conflict: a theoretical review and critique of conflict-related ERPs. International Journal of Psychophysiology, 93(3), 283-297. https://doi.org/10.1016/j.ijpsycho.2014.06.007

Leuthold, H., \& Sommer, W. (1999). ERP correlates of error processing in spatial S-R compatibility tasks. Clinical Neurophysiology, 110(2), 342-357. https://doi.org/10.1016/S1388-2457(98)00058-3

Lilienfeld, S. O. (2018). The multidimensional nature of psychopathy: Five recommendations for research. Journal of Psychopathology and Behavioral Assessment, 40(1), 79-85. https://doi.org/10.1007/s10862-018-9657-7

Lilienfeld, S.O., \& Widows, M.R. (2005). Psychopathic Personality Inventory-Revised (PPI-R) professional manual. Odessa, FL: Psychological Assessment Resources.

Maurer, J. M., Steele, V. R., Cope, L. M., Vincent, G. M., Stephen, J. M., Calhoun, V. D., \& Kiehl, K. A. (2016). Dysfunctional error-related processing in incarcerated youth with elevated psychopathic traits. Developmental Cognitive Neuroscience, 19, 7077. https://doi.org/10.1016/j.den.2016.02.006

Maurer, J. M., Steele, V. R., Edwards, B. G., Bernat, E. M., Calhoun, V. D., \& Kiehl, K. A. (2016). Dysfunctional error-related processing in female psychopathy. Social 
Performance Monitoring ERPs and Triarchic Psychopathy

Cognitive and Affective Neuroscience, 11(7), 1059-1068.

https://doi.org/10.1093/scan/nsv070

Meyer, A., Riesel, A., \& Proudfit, G. H. (2013). Reliability of the ERN across multiple tasks as a function of increasing errors. Psychophysiology, 50, 1220-1225. https://doi.org/10.1111/psyp.12132

Munro, G. E., Dywan, J., Harris, G. T., McKee, S., Unsal, A., \& Segalowitz, S. J. (2007a). Response inhibition in psychopathy: the frontal N2 and P3. Neuroscience Letters, 418(2), 149-153. https://doi.org/10.1016/j.neulet.2007.03.017

Munro, G. E., Dywan, J., Harris, G. T., McKee, S., Unsal, A., \& Segalowitz, S. J. (2007b). ERN varies with degree of psychopathy in an emotion discrimination task. Biological Psychology, 76(1-2), 31-42. https://doi.org/10.1016/j.biopsycho.2007.05.004

Nelson, L. D., Patrick, C. J., \& Bernat, E. M. (2011). Operationalizing proneness to externalizing psychopathology as a multivariate psychophysiological phenotype. Psychophysiology, 48(1), 64-72. https://doi.org/10.1111/j.1469$\underline{8986.2010 .01047 . \mathrm{x}}$

Nieuwenhuis, S., Aston-Jones, G., \& Cohen, J. D. (2005). Decision making, the P3, and the locus coeruleus-norepinephrine system. Psychological Bulletin, 131(4), 510. https://doi.org/10.1037/0033-2909.131.4.510

Nieuwenhuis, S., Ridderinkhof, K. R., Blom, J., Band, G. P., \& Kok, A. (2001). Errorrelated brain potentials are differentially related to awareness of response errors: Evidence from an antisaccade task. Psychophysiology, 38(5), 752-760. https://doi.org/10.1111/1469-8986.3850752

Nieuwenhuis, S., Yeung, N., Van Den Wildenberg, W., \& Ridderinkhof, K. R. (2003). Electrophysiological correlates of anterior cingulate function in a go/no-go task: 
Performance Monitoring ERPs and Triarchic Psychopathy

Effects of response conflict and trial type frequency. Cognitive, Affective, \& Behavioral Neuroscience, 3(1), 17-26. https://doi.org/10.3758/CABN.3.1.17

Nigbur, R., Ivanova, G., \& Stürmer, B. (2011). Theta power as a marker for cognitive interference. Clinical Neurophysiology, 122(11), 2185-2194.

https://doi.org/10.1016/j.clinph.2011.03.030

O'Connell, R. G., Dockree, P. M., Bellgrove, M. A., Kelly, S. P., Hester, R., Garavan, H., ... \& Foxe, J. J. (2007). The role of cingulate cortex in the detection of errors with and without awareness: A high-density electrical mapping study. European Journal of Neuroscience, 25(8), 2571-2579. https://doi.org/10.1111/j.1460-9568.2007.05477.x

Olvet, D. M., \& Hajcak, G. (2009). The stability of error-related brain activity with increasing trials. Psychophysiology, 46(5), 957-961. https://doi.org/10.1111/j.14698986.2009.00848.x

Overbeek, T. J., Nieuwenhuis, S., \& Ridderinkhof, K. R. (2005). Dissociable components of error processing: On the functional significance of the Pe vis-à-vis the ERN/Ne. Journal of Psychophysiology, 19(4), 319-329. https://doi.org/10.1027/0269$\underline{8803.19 .4 .319}$

Pasion, R., \& Barbosa, F. (2019). ERN as a transdiagnostic marker of the InternalizingExternalizing Spectrum: A dissociable meta-analytic effect. Neuroscience \& Biobehavioral Reviews, 103, 133-149.

https://doi.org/10.1016/j.neubiorev.2019.06.013

Pasion, R., Cruz, A. R., \& Barbosa, F. (2016). Dissociation of boldness and disinhibition psychopathic traits in ERN modulation. Personality and Individual Differences, 95, 6-10. https://doi.org/10.1016/j.paid.2016.02.017

Pasion, R., Fernandes, C., Pereira, M. R., \& Barbosa, F. (2018). Antisocial behaviour and psychopathy: Uncovering the externalizing link in the P3 modulation. Neuroscience 
Performance Monitoring ERPs and Triarchic Psychopathy

\& Biobehavioral Reviews, 91, 170-186.

https://doi.org/10.1016/j.neubiorev.2017.03.012

Patrick, C. J. (2010). Operationalizing the triarchic conceptualization of psychopathy: preliminary description of brief scales for assessment of boldness, meanness, and disinhibition. Unpublished manual. Tallahassee: Department of Psychology, Florida State University [Retrieved from]. https://doi.org/www.phenxtoolkit.org

Patrick, C. J. (2018). Cognitive and emotional processing in psychopathy. In: C. J. Patrick (Ed.), Handbook of psychopathy (2nd ed.) (pp. 422-455). New York: Guilford Press.

Patrick, C. J., \& Bernat, E. M. (2009). Neurobiology of psychopathy: A two-process theory. In G. G. Berntson \& J. T. Cacioppo (Eds.), Handbook of neuroscience for the behavioral sciences (pp. 1110-1131). New York: Wiley. https://doi.org/10.1002/9780470478509.neubb002057

Patrick, C. J., Bernat, E. M., Malone, S. M., Iacono, W. G., Krueger, R. F., \& McGue, M. (2006). P300 amplitude as an indicator of externalizing in adolescent males. Psychophysiology, 43(1), 84-92. https://doi.org/10.1111/j.1469$\underline{8986.2006 .00376 . x}$

Patrick, C. J., \& Drislane, L. E. (2015). Triarchic model of psychopathy: Origins, operationalizations, and observed linkages with personality and general psychopathology. Journal of Personality, 83(6), 627-643.

https://doi.org/10.1111/jopy.12119

Patrick, C. J., Durbin, C. E., \& Moser, J. S. (2012). Reconceptualizing antisocial deviance in neurobehavioral terms. Development and Psychopathology, 24(3), 1047-1071. https://doi.org/10.1017/S0954579412000533

Patrick, C. J., Fowles, D. C., \& Krueger, R. F. (2009). Triarchic conceptualization of psychopathy: Developmental origins of disinhibition, boldness, and meanness. 
Performance Monitoring ERPs and Triarchic Psychopathy

Development and Psychopathology, 21(3), 913-938.

https://doi.org/10.1017/S0954579409000492

Patrick, C. J., \& Hajcak, G. (2016). RDoC: Translating promise into progress.

Psychophysiology, 53(3), 415-424. https://doi.org/10.1111/psyp.12612

Patrick, C. J., Hicks, B. M., Krueger, R. F., \& Lang, A. R. (2005). Relations between psychopathy facets and externalizing in a criminal offender sample. Journal of Personality Disorders, 19(4), 339-356. https://doi.org/10.1521/pedi.2005.19.4.339

Patrick, C. J., Venables, N. C., Yancey, J. R., Hicks, B. M., Nelson, L. D., \& Kramer, M. D. (2013). A construct-network approach to bridging diagnostic and physiological domains: Application to assessment of externalizing psychopathology. Journal of Abnormal Psychology, 122(3), 902-916. https://doi.org/10.1037/a0032807

Perkins, E. R., Yancey, J. R., Drislane, L. E., Venables, N. C., Balsis, S., \& Patrick, C. J. (2017). Methodological issues in the use of individual brain measures to index trait liabilities: The example of noise-probe P3. International Journal of Psychophysiology, 111, 145-155. https://doi.org/10.1016/j.ijpsycho.2016.11.012

Polich, J. (2007). Updating P300: an integrative theory of P3a and P3b. Clinical Neurophysiology, 118(10), 2128-2148. https://doi.org/10.1016/j.clinph.2007.04.019

Poy, R., Segarra, P., Esteller, À., López, R., \& Moltó, J. (2014). FFM description of the triarchic conceptualization of psychopathy in men and women. Psychological Assessment, 26(1), 69-76. https://doi.org/10.1037/a0034642

Ridderinkhof, K. R., Ramautar, J. R., \& Wijnen, J. G. (2009). To PE or not to PE: A P3-like ERP component reflecting the processing of response errors. Psychophysiology, 46(3), 531-538. https://doi.org/10.1111/j.1469-8986.2009.00790.x

Riesel, A., Weinberg, A., Endrass, T., Meyer, A., \& Hajcak, G. (2013). The ERN is the ERN is the ERN? Convergent validity of error-related brain activity across different 
tasks. Biological Psychology, 93(3), 377-385.

https://doi.org/10.1016/j.biopsycho.2013.04.007

Ruchensky, J. R., Donnellan, M. B., \& Edens, J. F. (2018). Development and initial validation of the HEXACO-Triarchic scales. Psychological Assessment, 30(12), 1560-1566. https://doi.org/10.1037/pas0000609

Schulreich, S. (2016). Altered performance monitoring in psychopathy: A review of studies on action selection, error, and feedback processing. Current Behavioral Neuroscience Reports, 3(1), 19-27. https://doi.org/10.1007/s40473-016-0061-x

Schulreich, S., Pfabigan, D. M., Derntl, B., \& Sailer, U. (2013). Fearless Dominance and reduced feedback-related negativity amplitudes in a time-estimation task-Further neuroscientific evidence for dual-process models of psychopathy. Biological Psychology, 93(3), 352-363. https://doi.org/10.1016/j.biopsycho.2013.04.004

Skeem, J. L., Polaschek, D. L. L., Patrick, C. J., \& Lilienfeld, S. O. (2011). Psychopathic personality: Bridging the gap between scientific evidence and public policy. Psychological Science in the Public Interest, 12, 95-162. https://doi.org/10.1177/1529100611426706

Steele, V. R., Maurer, J. M., Bernat, E. M., Calhoun, V. D., \& Kiehl, K. A. (2016). Errorrelated processing in adult males with elevated psychopathic traits. Personality Disorders: Theory, Research, and Treatment, 7(1), 80-90. https://doi.org/10.1037/per0000143

Steinhauser, M., \& Yeung, N. (2012). Error awareness as evidence accumulation: effects of speed-accuracy trade-off on error signaling. Frontiers in human neuroscience, 6, 240. https://doi.org/10.3389/fnhum.2012.00240

Ullsperger, M., Fischer, A. G., Nigbur, R., \& Endrass, T. (2014). Neural mechanisms and temporal dynamics of performance monitoring. Trends in Cognitive Sciences, 18(5), 259-267. https://doi.org/10.1016/j.tics.2014.02.009 
Performance Monitoring ERPs and Triarchic Psychopathy

Van Noordt, S. J., Campopiano, A., \& Segalowitz, S. J. (2016). A functional classification of medial frontal negativity ERPs: Theta oscillations and single subject effects. Psychophysiology, 53(9), 1317-1334. https://doi.org/10.1111/psyp.12689

Van Veen, V., \& Carter, C. S. (2002). The timing of action-monitoring processes in the anterior cingulate cortex. Journal of Cognitive Neuroscience, 14(4), 593-602. https://doi.org/10.1162/08989290260045837

Venables, N. C., Foell, J., Yancey, J. R., Kane, M. J., Engle, R. W., \& Patrick, C. J. (2018). Quantifying inhibitory control as externalizing proneness: A cross-domain model. Clinical Psychological Science, 6(4), 561-580.

\section{https://doi.org/10.1177/2167702618757690}

Venables, N. C., \& Patrick, C. J. (2014). Reconciling discrepant findings for P3 brain response in criminal psychopathy through reference to the concept of externalizing proneness. Psychophysiology, 51(5), 427-436. https://doi.org/10.1111/psyp.12189

Vilà-Balló, A., Hdez-Lafuente, P., Rostan, C., Cunillera, T., \& Rodríguez-Fornells, A. (2014). Neurophysiological correlates of error monitoring and inhibitory processing in juvenile violent offenders. Biological Psychology, 102, 141-152. https://doi.org/10.1016/j.biopsycho.2014.07.021

Weinberg, A., Dieterich, R., \& Riesel, A. (2015). Error-related brain activity in the age of RDoC: A review of the literature. International Journal of Psychophysiology, 98(2), 276-299. https://doi.org/10.1016/j.ijpsycho.2015.02.029

Wilcox, R. R. (2012). Modern statistics for the social and behavioral sciences: A practical introduction. New York: Springer.

Yeung, N., Botvinick, M. M., \& Cohen, J. D. (2004). The neural basis of error detection: Conflict monitoring and the error-related negativity. Psychological Review, 111(4), 931-959. https://doi.org/10.1037/0033-295X.111.4.931 
Performance Monitoring ERPs and Triarchic Psychopathy

Young, S. E., Friedman, N. P., Miyake, A., Willcutt, E. G., Corley, R. P., Haberstick, B. C., \& Hewitt, J. K. (2009). Behavioral disinhibition: Liability for externalizing spectrum disorders and its genetic and environmental relation to response inhibition across adolescence. Journal of Abnormal Psychology, 118(1), 117-130. https://doi.org/10.1037/a0014657 
Performance Monitoring ERPs and Triarchic Psychopathy

\section{Author notes}

We are grateful to Dr. Noah C. Venables for the scholarly input provided on a previous draft of this article.

Funding: This work was supported by the MINECO (Spain) [grant number: PSI2011-22559], the MINECO/FEDER (Spain) [grant number: PSI2015-66798-P], and the Universitat Jaume I [grant number: P1·1B2013-12]. The first author was supported by the MINECO (Spain) [grant numbers: BES-2012-053791, EEBB-I-14-08797] and the Generalitat Valenciana and European Social Fund [grant number: APOSTD/2018/A/068]. The third author was supported by grant W911NF-14-1-0018 from the US Army. The content of this paper is solely the responsibility of the authors and does not necessarily reflect the official views of the U.S. Government, Department of Defense, Department of the Army, Department of Veterans Affairs, or U.S. Recruiting Command.

Conflict of interest: The authors have no conflict of interest to report

Address reprint requests to: Pablo Ribes-Guardiola, PhD. E-mail: pribes@uji.es 


\section{Footnotes}

1. The grand-average plots shown in Figure 1 include waveforms for the correct response condition of each task (CRN, Pc), the go condition of the go/no-go task (go N2, go P3), and the congruent condition of the flanker task (congruent $\mathrm{N} 2$, congruent $\mathrm{P}$ 3) - each quantified using the time windows and electrode clusters already described - in order to illustrate within-subject condition effects for the different ERP measures from the two tasks (see Supplementary materials).

2. Our choice to quantify the ERN and Pe in the flanker task collapsing across incongruent and congruent error trials was based on prior research that has likewise used this approach to examine the covariance between error related ERPs across go/no-go and flanker tasks (e.g., Burwell et al., 2016; Riesel et al., 2013). Supplemental analyses carried out for ERN and Pe amplitudes based on incongruent error trials only revealed the same pattern of results reported in the main text.

3. In addition, we tested for possible interactions between gender and the triarchic dimensions by means of hierarchical regression analyses in which gender and TriPM scale scores were entered concurrently as predictors at Step 1, and the three gender $\mathrm{x}$ TriPM scale score interactions were entered at Step 2. Because these analyses did not reveal a significant increase in $R^{2}$ in any case at Step $2($ all $p$ s $>.05)$ - indicating no significant effect of gender on relations of TriPM scale scores with response measures - only results from the hierarchical regression models controlling for gender at Step 1 are reported in the Results section. Furthermore, and based on the configural nature of psychopathic personality embodied in the triarchic model (Lilienfeld, 2018; 
Performance Monitoring ERPs and Triarchic Psychopathy

Patrick et al., 2009; Skeem et al., 2011), we also tested for potential interactions between the orthogonal dimensions of boldness and disinhibition in predicting task response measures by means of hierarchical regression analyses. Results of these analyses did not reveal a significant interaction between boldness and disinhibition in predicting any task response measure $\left(\Delta F_{S}(1,137)<1.40, p \mathrm{~s}>.24, \Delta R^{2}<.010\right)$.

4. As noted in the in the Method section, P3 response was quantified for each task using data from a cluster of midline centroparietal electrode sites. At the request of an anonymous reviewer, we evaluated whether the associations for go/no-go and flanker P3 amplitude with TriPM Disinhibition scores were similar or different at midline frontocentral sites (i.e., those used in quantifying ERN and N2). For both go/no-go and flanker P3 response, we found partial $r \mathrm{~s}$ (analogous to $\beta \mathrm{s}$ at Step 2 of the reported regression models) to be highly similar for frontocentral sites (= -.17 and -.21 , respectively) and centroparietal sites $(=-.18$ and -.26$)$.

5. Given that the ERN has been found to covary with error probability (Fischer, Klein, \& Ullsperger, 2017), we conducted a supplemental analysis testing for the possibility that contrasting associations between no-go and flanker task variants of the ERN in relation to trait disinhibition were accounted for by differences in error rates across tasks. Of particular interest was whether TriPM Disinhibition scores were still associated with no-go ERN amplitude after accounting for no-go accuracy rate, which showed a significant bivariate association with no-go ERN ( $r=-.17, p=.042)$; the counterpart association for flanker ERN was not significant $(r=-.05, p=.569)$. A partial correlation analysis revealed that TriPM Disinhibition scores were still associated with 
no-go ERN amplitude when controlling for no-go task accuracy and gender (partial $r=.18, p=.031$ ). Thus, our finding of reduced no-go ERN (but not Flanker ERN) amplitude in relation to trait disinhibition is not accounted for by the differing error rates across the two tasks, and may instead reflect task specific aspects of the no-go ERN.

6. At the request of an anonymous reviewer, we performed supplemental analyses examining associations between the ERP measures from the two tasks and total scores on the TriPM (i.e., sum of item scores across subscales). At the bivariate level, TriPM total scores were associated only with reduced (i.e., less negative) no-go ERN amplitude $(r=.18, p=.032)$. In partial correlations controlling for gender, TriPM total scores showed a significant positive association with no-go ERN amplitude (partial $r=.18, p=.029$ ), indicating reduced ERN with higher TriPM total scores, and significant negative associations with No-go P3, Incongruent P3, No-go Pe, and flanker Pe (partial $r \mathrm{~s}=-.17,-.24,-.19$, and -.23 , respectively, $p \mathrm{~s}=.049, .004, .023$, and .007), indicating reduced amplitude of response for each of these ERP measures with higher TriPM total scores. 


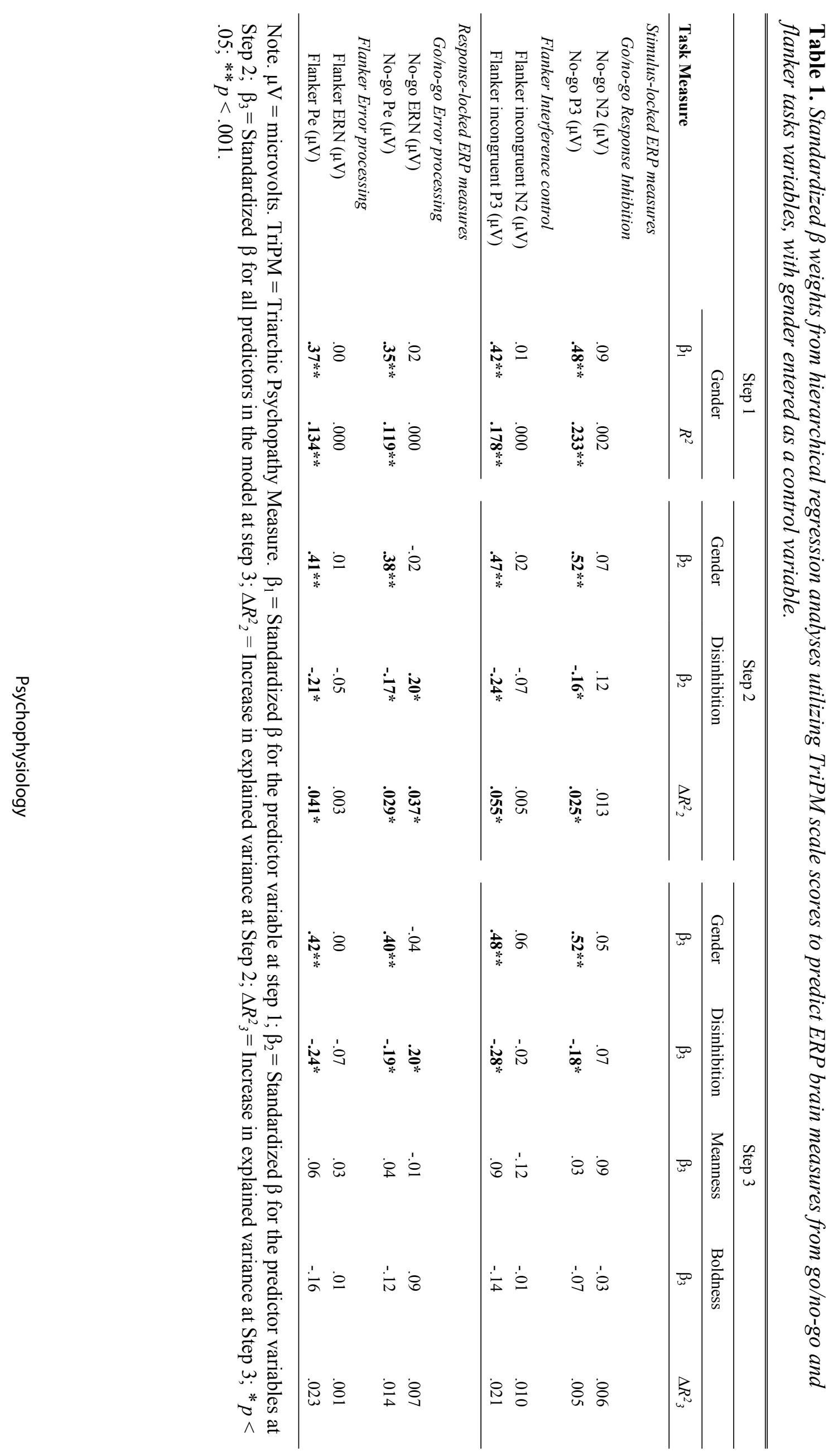




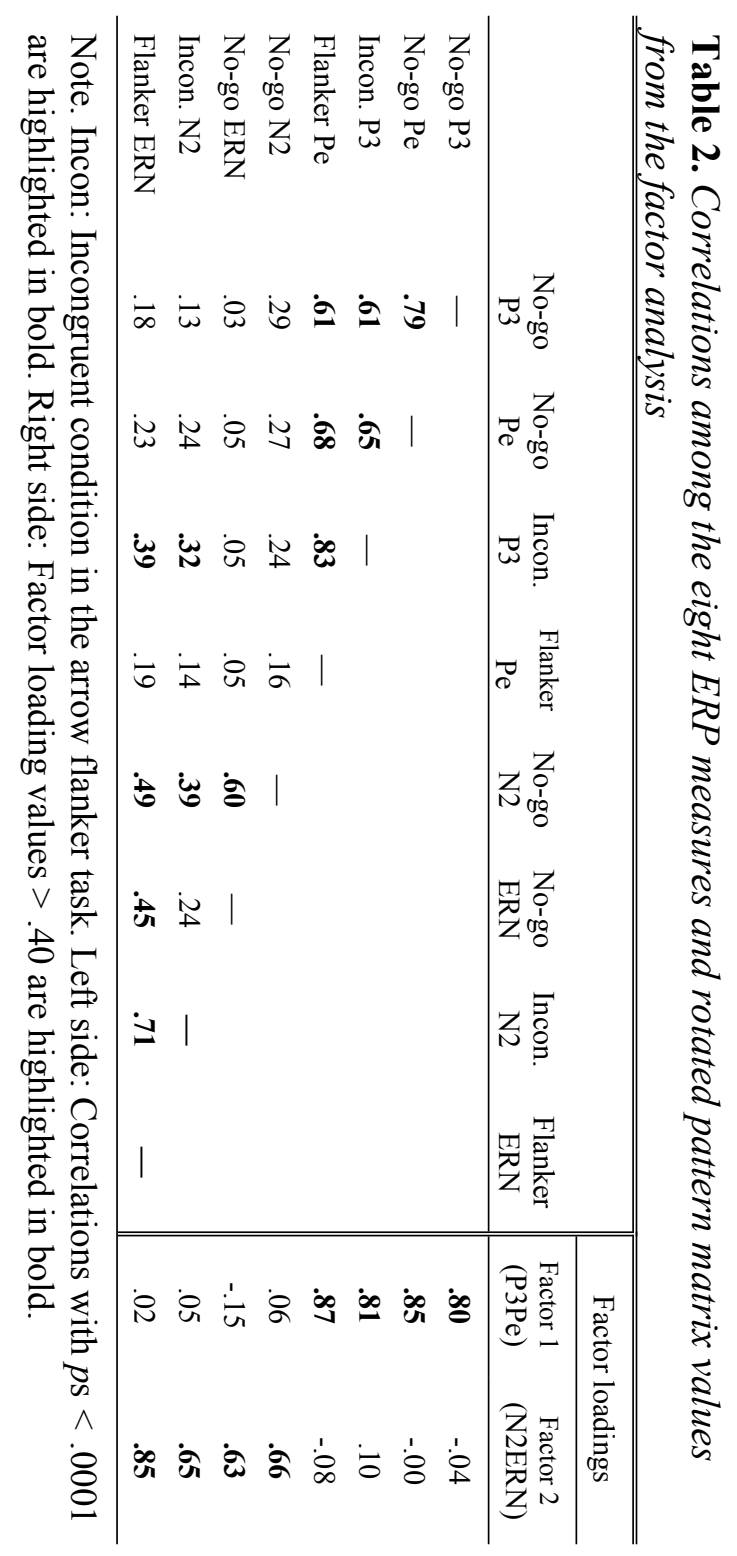




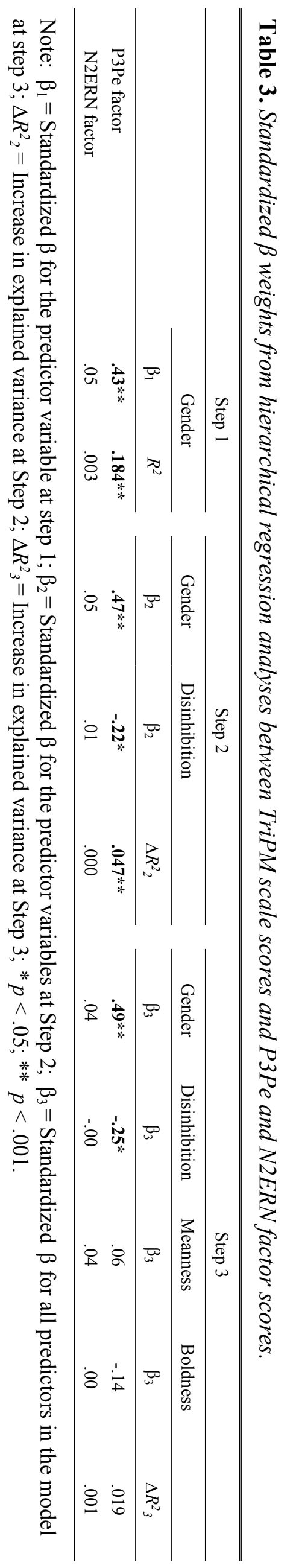


Performance Monitoring ERPs and Triarchic Psychopathy

\section{Figure Captions}

Figure 1. Grand average waveforms and topographic map distributions for ERP components derived from the go/no-go task (left side) and the arrow flanker task (right side). (A) Grand average stimulus-locked ERP waveforms for correct-response Go (Go; dotted lines) and successful inhibition (no-go; solid lines) trials in the go/no-go task at the frontocentral electrode cluster. (B) Go N2 and no-go N2 scalp distribution maps for the 250-350 ms poststimulus time window. (C) Grand average stimulus-locked ERP waveforms for correct-response Congruent (dotted lines) and Incongruent (solid lines) trials in the arrow flanker task at the frontocentral electrode cluster. (D) Congruent N2 and Incongruent N2 scalp distribution for the 250-350 ms poststimulus time window. (E) Grand average stimulus-locked ERP waveforms for correct-response Go (Go; dotted lines) and successful inhibition (No-go; solid lines) trials in the go/no-go task at the centroparietal electrode cluster. (F) Go P3 and no-go P3 scalp distribution for the 350$550 \mathrm{~ms}$ poststimulus time window. (G) Grand average stimulus-locked ERP waveforms for correct-response Congruent (dotted lines) and Incongruent (solid lines) trials in the arrow flanker task at the centroparietal electrode cluster. (H) Congruent P3 and Incongruent P3 scalp distribution for the 300-550 ms poststimulus window. (I) Grand average response-locked ERP waveforms for correct Go (CRN; dotted lines) and false alarm (ERN; solid lines) trials in the go/no-go task at the frontocentral electrode cluster. (J) CRN and ERN scalp distribution for the $0-100 \mathrm{~ms}$ postresponse time window in the go/no-go task. (K) Grand average response-locked ERP waveforms for correct (CRN; dotted lines) and error (ERN; solid lines) trials in the arrow flanker task at the frontocentral electrode cluster. (L) $\mathrm{CRN}$ and ERN scalp distribution for the $0-100 \mathrm{~ms}$ postresponse time window in the arrow flanker task. (M) Grand average responselocked ERP waveforms for correct Go (Pc; dotted lines) and false alarm (Pe; solid lines) trials in the go/no-go task at the centroparietal electrode cluster. (N) Pc and Pe scalp 
Performance Monitoring ERPs and Triarchic Psychopathy

distribution for the 150-400 ms postresponse time window in the go/no-go task. (O) Grand average response-locked ERP waveforms for correct (Pc; dotted lines) and error (Pe; solid lines) trials in the arrow flanker task at the centroparietal electrode cluster. (P) Pc and Pe scalp distribution for the 150-400 ms postresponse time window in the arrow flanker task.

Figure 2. Grand average waveforms and topographic map distributions for participants scoring low $(n=70)$ versus high $(n=72)$ on the TriPM Disinhibition scale (DIS; median-split groups, with men and women grouped according to gender-specific medians). (A) Grand average stimulus-locked ERP waveforms for correct-response Go (Go; dotted lines) and successful inhibition (No-go; solid lines) trials in the go/no-go task at the centroparietal electrode cluster. (B) No-go P3 scalp distribution maps for the 350-550 ms poststimulus time window. (C) Grand average stimulus-locked ERP waveforms for correct-response Congruent (dotted lines) and Incongruent (solid lines) trials in the arrow flanker task at the centroparietal electrode cluster. (D) Incongruent P3 scalp distribution for the $300-550$ ms poststimulus time window. (E) Grand average response-locked ERP waveforms for correct Go (CRN; dotted lines) and false alarm (ERN; solid lines) trials in the go/no-go task for the frontocentral electrode cluster. (F) ERN scalp distribution for the $0-100 \mathrm{~ms}$ postresponse time window in the go/no-go task. (G) Grand average response-locked ERP waveforms for correct (CRN; dotted lines) and error (ERN; solid lines) trials in the arrow flanker task at the frontocentral electrode cluster. (H) ERN scalp distribution for the 0-100 ms postresponse time window in the arrow flanker task. (I) Grand average response-locked ERP waveforms for correct Go (Pc; dotted lines) and false alarm (Pe; solid lines) trials in the go/no-go task at the centroparietal electrode cluster. (J) Pe scalp distribution for the 150-400 ms postresponse time window in the go/no-go task. (K) Grand average response-locked 
Performance Monitoring ERPs and Triarchic Psychopathy

ERP waveforms for correct (Pc; dotted lines) and error (Pe; solid lines) trials in the arrow flanker task at the centroparietal electrode cluster. (L) Pe scalp distribution for the 150-400 ms postresponse time window in the arrow flanker task. 
Go/No-go task

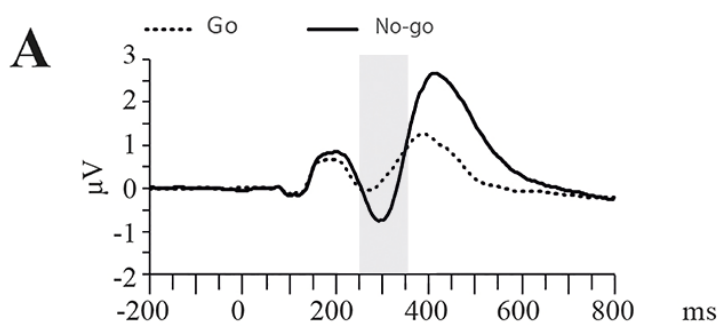

B

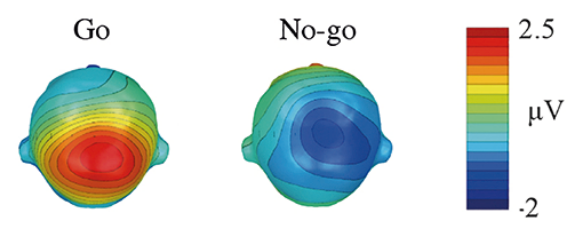

Psyવw2000siology Flanker task
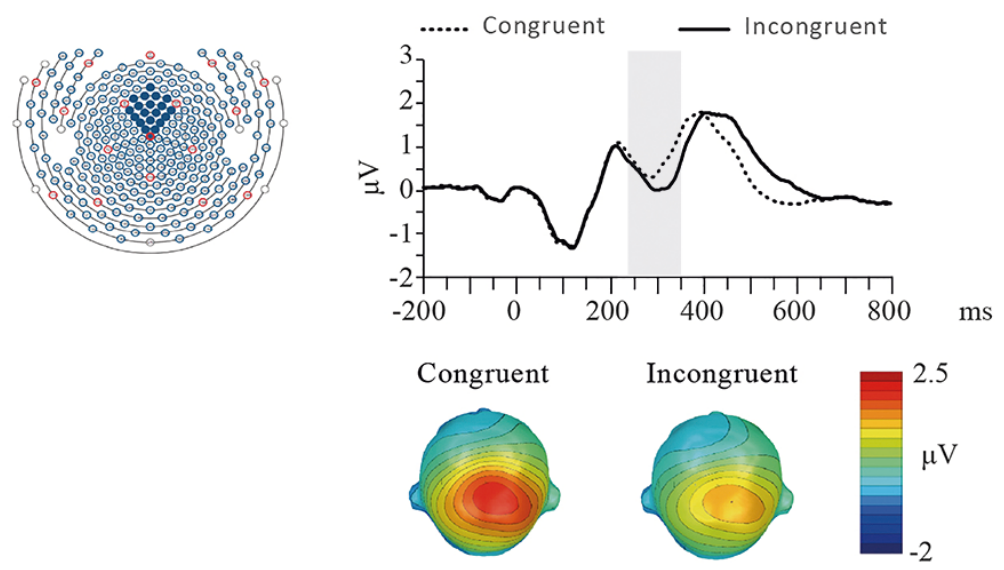

ms

C

\section{P300}
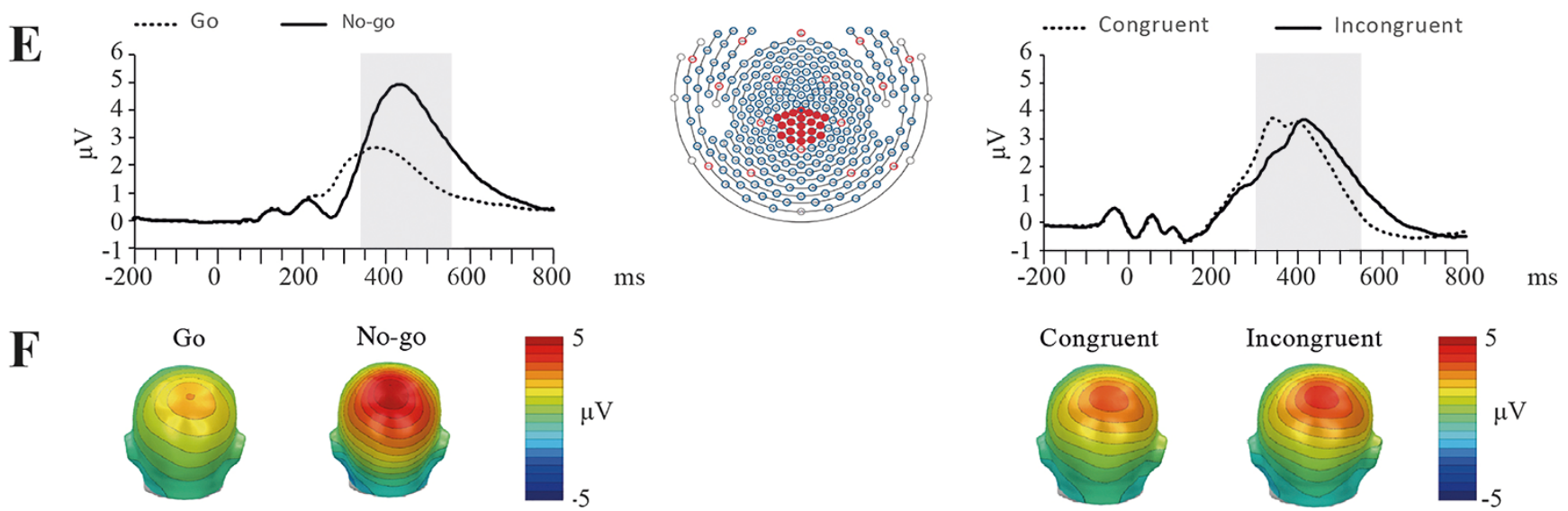

\section{ERN}

I
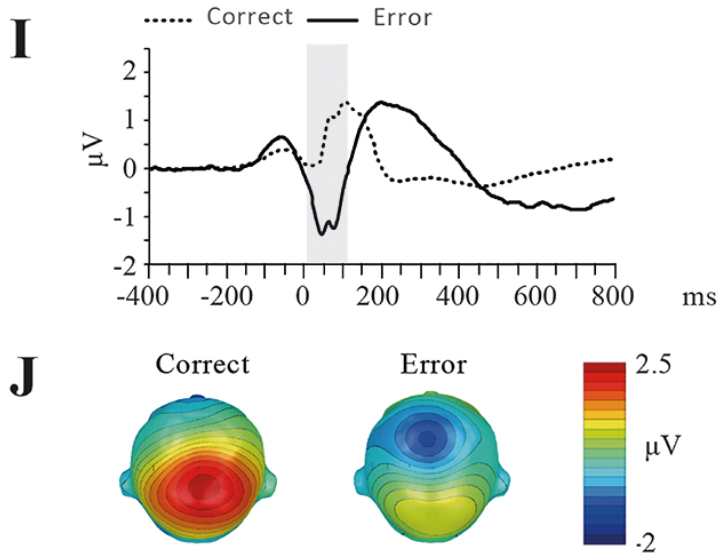

M

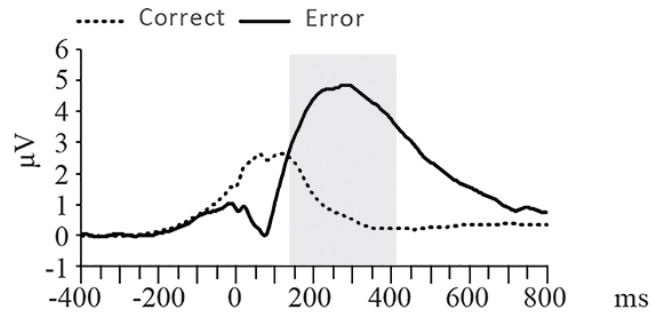

$\mathbf{N}$

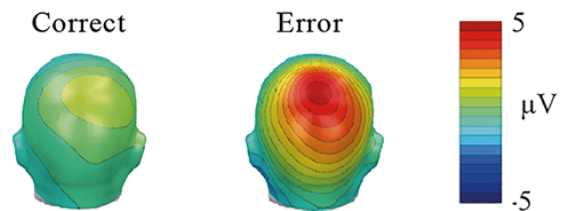

Psychophysiology
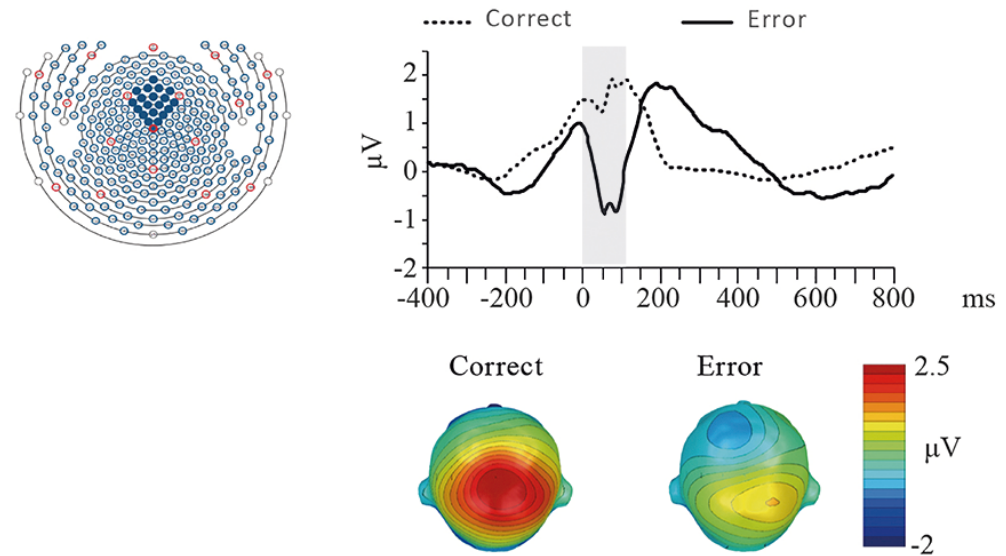

\section{Pe}
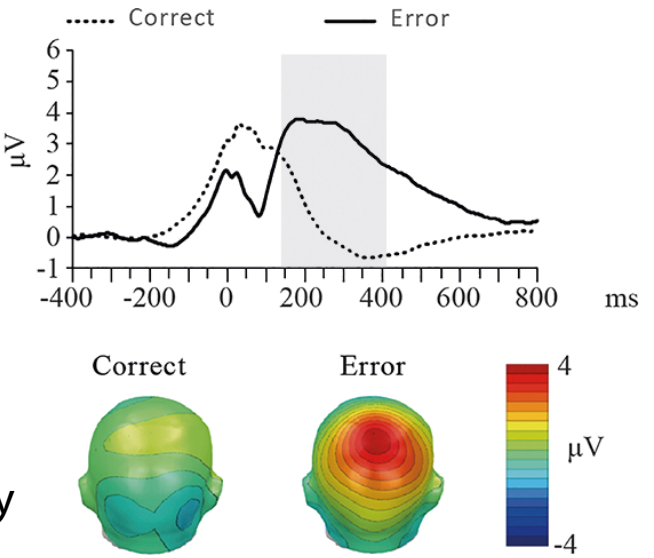

K

O

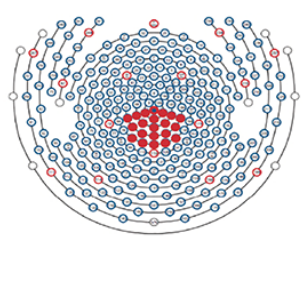



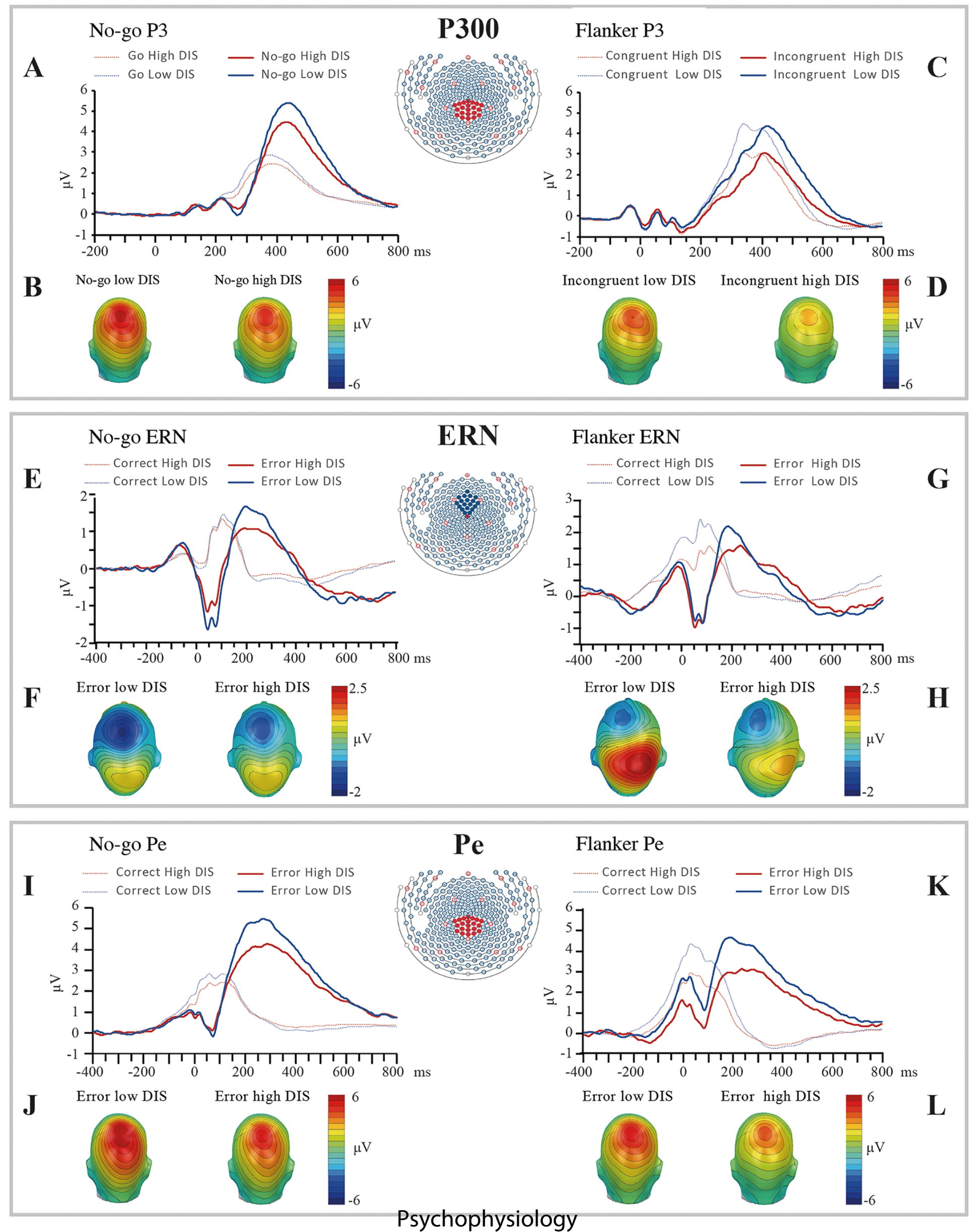
PERFORMANCE MONITORING ERPS AND TRIARCHIC PSYCHOPATHY - 1 SUPPLEMENT

Electrocortical Measures of Performance Monitoring from Go/No-Go and Flanker

Tasks: Differential Relations with Trait Dimensions of the Triarchic Model of

Psychopathy

Supplementary Materials

\section{$\underline{\text { Contents }}$}

1. Descriptive Statistics for the Triarchic Psychopathy Measure.

p. 3. Supplementary Table S1. TriPM scale score Means (M), Standard Deviations (SD), and range for participants in the current study.

2. Construct Validity of the Triarchic Psychopathy Measure

p. 5. Supplementary Table S2. Relationships between TriPM scale scores and criterion measures of personality in the overall study sample $(N=161)$ : Bivariate correlations $(r)$ and standardized beta weights $(\beta)$ from multiple regression analyses.

3. Behavioral and ERP Measures: Condition Effects for the Final Study Sample $(N=142)$ and Associations with Triarchic Dimensions

\subsection{Behavioral Effects in the Go/No-go Task}

p. 7. Supplementary Table S3. Split-half Reliabilities, Means (M), and Standard Deviations (SD) for behavioral measures in the go/no-go task. p. 8. Supplementary Table S4. Relationships between TriPM scale scores and behavioral measures in the go/no-go task.

3.2. Behavioral Effects in the Arrow Flanker Task

p. 9. Supplementary Table S5. Split-half Reliabilities, Means (M), and Standard Deviations (SD) for behavioral measures in the arrow flanker task.

p. 9. Supplementary Table S6. Relationships between TriPM scale scores and behavioral measures in the arrow flanker task. 
PERFORMANCE MONITORING ERPS AND TRIARCHIC PSYCHOPATHY -

SUPPLEMENT

3.3. Stimulus-Locked ERP Effects: Response Inhibition and Flanker Interference

p. 10. Supplementary Table S7. Split-half Reliabilities, Means (M), and Standard Deviations (SD) for Stimulus-locked ERPs in the go/no-go and the arrow flanker task.

p. 13. Supplementary Table S8. Relationships between TriPM scale scores and Stimulus-Locked ERPs in both the go/no-go and the arrow flanker task.

3.4. Response-Locked ERP Effects: Error Processing in the Go/no-go and the Arrow Flanker Task

p. 14. Supplementary Table S9. Split-half Reliabilities, Means (M), and Standard Deviations (SD) for Response-locked ERPs in the go/no-go and the arrow flanker task.

p.15. Supplementary Table S10. Relationships between TriPM scale scores and Response-Locked ERPs in both the go/no-go and the arrow flanker task.

4. Exploratory Investigation of Associations between Triarchic Dimensions and Difference Scores for Study ERP Measures.

p. 17. Supplementary Table S11. Associations between TriPM scale scores and condition-difference scores for ERP measures.

\section{Supplementary References}

p. $19-22$ 
PERFORMANCE MONITORING ERPS AND TRIARCHIC PSYCHOPATHY - 3 SUPPLEMENT

\section{Descriptive statistics for the Triarchic Psychopathy Measure}

The Supplementary Table S1 shows TriPM scale score $M s$, SDs, and ranges for the sample as a whole, and for women and men separately, along with independent samples $t$-tests for significant gender differences in TriPM scale scores.

As can be seen in Table S1, independent samples $t$-tests revealed that men scored significantly higher than women on all TriPM scales, consistent with prior work using the Spanish adaptation of the TriPM (Esteller, Poy, \& Moltó, 2016; Poy, Segarra, Esteller, López, \& Moltó, 2014).

Supplementary Table S1. TriPM scale score Means (M), Standard Deviations (SD), and range for participants in the current study.

\begin{tabular}{|c|c|c|c|c|c|c|c|c|}
\hline & \multicolumn{2}{|c|}{$\begin{array}{l}\text { All participants } \\
\qquad(n=161)\end{array}$} & \multicolumn{2}{|c|}{$\begin{array}{l}\text { Women } \\
(n=114)\end{array}$} & \multicolumn{2}{|c|}{$\begin{array}{c}\text { Men } \\
(n=47)\end{array}$} & \multicolumn{2}{|c|}{ Women vs. Men difference } \\
\hline & $M(S D)$ & Range & $M(S D)$ & Range & $M(S D)$ & Range & $t$ & $p$ \\
\hline Boldness & $29.73(8.44)$ & $7-48$ & $28.33(7.64)$ & $7-45$ & $33.13(9.36)$ & $8-48$ & -3.38 & .000 \\
\hline Meanness & $10.11(6.33)$ & $0-31$ & $8.82(5.60)$ & $0-30$ & $13.23(6.93)$ & $4-31$ & -4.28 & .000 \\
\hline Disinhibition & $14.64(7.67)$ & $0-39$ & $13.78(7.29)$ & $0-33$ & $16.72(8.22)$ & $0-39$ & -2.24 & .026 \\
\hline Total score & $54.48(15.68)$ & $18-106$ & $50.94(14.04)$ & $18-98$ & $63.08(16.29)$ & $32-106$ & -4.76 & .000 \\
\hline
\end{tabular}

Note. TriPM = Triarchic Psychopathy Measure. Independent samples $t$-tests for evaluation of gender differences have $159 d f$. Significant gender differences are in bold font.

\section{Construct validity of the Triarchic Psychopathy Measure}

The validity of the TriPM scale scores in relation to other self-report measures of personality administered to the overall study sample $(N=161)$ was evaluated by means of correlational analyses as well as by multiple regression analyses in which the three TriPM scale scores were entered concurrently as predictors of each personality measure. The standardized beta weights from these analyses are reported as indices of the unique contribution of each TriPM scale score to the prediction of each personality criterion measure. Statistical analyses were carried out using SPSS General linear model 
PERFORMANCE MONITORING ERPS AND TRIARCHIC PSYCHOPATHY SUPPLEMENT

software (Version 23, SPSS Inc. Chicago, IL). Results from these analyses are shown in the Supplementary Table S2.

Consistent with the theoretical conceptualization of boldness as involving high levels of emotional stability and immunity to stress, high social assurance and dominance, and venturesomeness (Patrick \& Drislane, 2015; Patrick, Fowles, \& Krueger, 2009), TriPM Boldness scores were strongly and negatively related to measures of neuroticism, anxiety, and sensitivity to punishment, and positively related to extraversion, openness to experience, and sensitivity to reward. TriPM Meanness scores were mainly related to agreeableness (negatively), consistent with its formulation as a phenotypic construct reflecting low levels of social connectedness and high antagonism (Patrick \& Drislane, 2015; Patrick et al., 2009). Finally, TriPM Disinhibition scores were strongly and positively related to indices of impulsivity and sensitivity to reward, as well as to neuroticism and anxiety, and negatively to conscientiousness, consistent with the view of this triarchic trait as encompassing unrestrained externalizing tendencies along with dysregulated negative affect (Patrick \& Drislane, 2015; Patrick et al., 2009).

Collectively, these results are consistent with prior published evidence for the construct validity of the TriPM scales in relation to measures of normal-range and pathological personality traits in both incarcerated and non-incarcerated samples from different countries (e.g., Almeida et al., 2015; Esteller et al., 2016; Poy et al., 2014; Sellbom \& Phillips, 2013; Sica et al., 2015; Stanley, Wygant, \& Sellbom, 2013; Strickland, Drislane, Lucy, Krueger, \& Patrick, 2013; van Dongen, Drislane, Nijman, Soe-Agnie, \& van Marle, 2017; see Patrick \& Drislane, 2015 for a review), and provide evidence for the clinical relevance of scores on the TriPM scales in the current study sample. 
SUPPLEMENT

Supplementary Table S2. Relationships between TriPM scale scores and criterion measures of personality in the overall study sample $(N=161)$ : Bivariate correlations $(r)$ and standardized beta weights $(\beta)$ from multiple regression analyses.

\begin{tabular}{|c|c|c|c|c|c|c|c|}
\hline \multirow[b]{3}{*}{ Personality Measures } & \multicolumn{7}{|c|}{ TriPM } \\
\hline & \multicolumn{2}{|c|}{ Boldness } & \multicolumn{2}{|c|}{ Meanness } & \multicolumn{2}{|c|}{ Disinhibition } & \multirow[b]{2}{*}{$R^{2}$} \\
\hline & $r$ & $\beta$ & $r$ & $\beta$ & $r$ & $\beta$ & \\
\hline \multicolumn{8}{|l|}{ NEO-FFI } \\
\hline Neuroticism & $-.51 *$ & $-.48 *$ & -.02 & $-.21 *$ & $.41 *$ & $.53 *$ & $.46^{*}$ \\
\hline Extraversion & $.44 *$ & $.47 *$ & -.00 & -.14 & .00 & .07 & $.21^{*}$ \\
\hline Openness & $.39 *$ & $.44^{*}$ & -.11 & $-.28 *$ & .00 & .15 & $.21 *$ \\
\hline Agreeableness & $-.25 *$ & -.16 & $-.51^{*}$ & $-.46^{*}$ & $-.28 *$ & -.03 & $.29 *$ \\
\hline Conscientiousness & .12 & .13 & $-.25^{*}$ & -.02 & $-.49 *$ & $-.48 *$ & $.26^{*}$ \\
\hline STAI-T & $-.57 *$ & $-.56^{*}$ & .01 & -.08 & $.34 *$ & $.39 *$ & $.45^{*}$ \\
\hline \multicolumn{8}{|l|}{ SPSRQ } \\
\hline SP & $-.60 *$ & $-.59 *$ & -.13 & -.06 & .06 & .10 & $.36^{*}$ \\
\hline SR & $.34 *$ & $.31 *$ & $.37 *$ & .10 & $.44 *$ & $.39 *$ & $.31 *$ \\
\hline BIS-11 Total Score & .17 & .17 & $.35^{*}$ & -.04 & $.65^{*}$ & $.67 *$ & $.44^{*}$ \\
\hline Attentional Impulsiveness & -.07 & -.08 & $.24 *$ & -.01 & $.50 *$ & $.51 *$ & $.26^{*}$ \\
\hline Motor Impulsiveness & .16 & .17 & $.26^{*}$ & -.08 & $.52 *$ & $.56^{*}$ & $.30 *$ \\
\hline Non-Planning Impulsiveness & $.24 *$ & $.24 *$ & $.28 *$ & -.00 & $.43 *$ & $.43 *$ & $.24 *$ \\
\hline
\end{tabular}

Note : NEO-FFI = NEO Five Factor Inventory (Costa \& McCrae, 1992); STAI-T $=$ Trait scale from the State-Trait Anxiety Inventory (Spielberger, Gorsuch, \& Lushene, 1970); SPSRQ = Sensitivity to Punishment (SP) and Sensitivity to Reward (SR) Questionnaire (Torrubia, Ávila, Moltó, \& Caseras, 2001); BIS-11 = Barratt Impulsiveness Scale - Version 11 (Patton, Stanford, \& Barratt, 1995). ${ }^{*} p<.005$

\section{Behavioral and ERP measures: Condition effects for the final study sample $(N$ $=142$ ) and associations with triarchic dimensions}

In order to corroborate the validity of the task procedures employed in the current study, we provide evidence here for replication of previously reported within-subject condition effects for behavioral and ERP variables in both the go/no-go and the flanker task.

Paired samples $t$-tests were used to test for differences in accuracy and reaction time (RT) between go and no-go trials in the go/no-go task, and between congruent and incongruent trials in the flanker task. Reaction time variability (i.e., within-subjects SD for RT scores on correct trials) and the posterror slowing effect (PES; Rabbit, 1966), 
SUPPLEMENT

defined as the difference in RT following error trials relative to correct trials, were also examined in both tasks.

The same analytic strategy was used to test for a pattern of differential brain reactivity in the conditions of interest in both tasks: error vs. correct trials in both tasks (for response-locked ERPs), no-go vs. go trials (for stimulus-locked ERPs in the go/nogo task), and incongruent vs. congruent trials (for stimulus-locked ERPs in the flanker task). Correct-response counterparts to the ERN (CRN) and the Pe (Pc), as well as ERPs from the Go condition in the go/no-go task (go N2; go P3) and the Congruent condition in the arrow flanker task (congruent N2; congruent P3), were quantified using the same baseline intervals, time windows, and scalp recoding sites described in the main article. Grand-average waveforms and scalp distributions for these ERPs are shown in Figure 1 of the main article.

Reliabilities for these additional behavioral and ERP measures were computed using split-half (odd versus even trials) correlations adjusted for attenuation using the Spearman-Brown prophecy formula.

Descriptive statistics for the sample as a whole $(n=142)$, and for gender subgroups separately, together with independent samples t-tests for significant gender differences on each task response measure are presented in the following sections. Finally, bivariate associations of these additional behavioral and ERP variables with TriPM scale scores were examined by means of the Pearson correlation coefficient $(r)$ and by partial correlations controlling for gender $(0=$ female; $1=$ male $)$.

\subsection{Behavioral effects in the go/no-go task}

Supplementary Table S3 shows descriptive statistics for the behavioral variables extracted from the go/no-go task. Split half reliability estimates were high $(>.87)$ for all behavioral measures. Independent samples $t$-tests revealed that women participants 

SUPPLEMENT

were more accurate and responded slower to go stimuli than men participants, and also showed longer RT on correct go responses after error trials (see Table S3). As for within-subjects condition effects, paired sample $t$-tests revealed that participants were more accurate on go trials than on no-go trials, $t(141)=28.16, p<.0001$, and responded more slowly on correct (go) trials than on error (no-go) trials, $t(141)=$ 31.57, $p<.0001$. A significant posterror slowing (PES) effect was observed, with longer RTs for correct go responses following errors than after successful inhibitions (correct rejections) to no-go stimuli, $t(141)=2.07, p<.05$ (See Table S3).

Supplementary Table S3. Split-half Reliabilities, Means (M), and Standard Deviations (SD) for behavioral measures in the go/no-go task.

\begin{tabular}{|c|c|c|c|c|c|c|c|c|c|}
\hline \multirow[b]{2}{*}{ Go/no-go Behavioral Measures } & \multirow[t]{2}{*}{$\begin{array}{l}\text { Split-half } \\
\text { Reliability }\end{array}$} & \multicolumn{2}{|c|}{$\begin{array}{l}\text { All Participants } \\
\quad(n=142)\end{array}$} & \multicolumn{2}{|c|}{$\begin{array}{l}\text { Women } \\
(n=101)\end{array}$} & \multicolumn{2}{|c|}{$\begin{array}{c}\text { Men } \\
(n=41)\end{array}$} & \multicolumn{2}{|c|}{$\begin{array}{c}\text { Women vs } \\
\text { Men difference }\end{array}$} \\
\hline & & $M$ & $S D$ & $M$ & $S D$ & $M$ & $S D$ & $t$ & $p$ \\
\hline Go Accuracy (\%) & .98 & 97.16 & 3.92 & 97.55 & 3.54 & 96.20 & 4.64 & 1.87 & .063 \\
\hline No-go Accuracy (\%) & .96 & 63.49 & 15.35 & 65.51 & 14.55 & 58.51 & 16.29 & 2.51 & .013 \\
\hline Go Correct RT (ms) & .99 & 331 & 34 & 334 & 32 & 321 & 39 & 2.19 & .031 \\
\hline Error RT (ms) & .89 & 282 & 28 & 284 & 29 & 277 & 27 & 1.31 & .193 \\
\hline Go RT variability (ms) & .96 & 92 & 19 & 93 & 17 & 91 & 20 & 0.61 & .541 \\
\hline Post Error RT (ms) & .94 & 314 & 45 & 320 & 44 & 300 & 43 & 2.48 & .014 \\
\hline Post Correct Rejection RT (ms) & .96 & 308 & 40 & 311 & 38 & 301 & 45 & 1.26 & .207 \\
\hline Post Error Slowing (ms) & .87 & 6 & 35 & 9 & 36 & -1 & 32 & 1.66 & .100 \\
\hline
\end{tabular}

Note. $\mathrm{RT}=$ Reaction time. $\mathrm{ms}=$ milliseconds. Independent samples $t$-tests for evaluation of gender differences have $140 d f$. Significant gender differences are shown in bold font.

Results from exploratory analyses testing for associations between TriPM scale scores and behavioral measures from the go/no-go task are reported in Supplementary Table S4. TriPM Meanness scores were negatively related to no-go accuracy, however, this association was non-significant when controlling for gender. TriPM Boldness scores only showed a significant bivariate inverse relationship with Post Error RT, which was non-significant after controlling for gender. Finally, TriPM Disinhibition scores evidenced a significant positive relationship with go trial RT variability, but only 

SUPPLEMENT

after controlling for gender. No other significant relationships were found (all $p \mathrm{~s}>.05$; see Table S4).

Supplementary Table S4. Relationships between TriPM scale scores and behavioral measures in the go/no-go task.

\begin{tabular}{|c|c|c|c|c|c|c|}
\hline \multirow[b]{2}{*}{ Go/no-go Behavioral Measures } & \multicolumn{2}{|c|}{ Boldness } & \multicolumn{2}{|c|}{ Meanness } & \multicolumn{2}{|c|}{ Disinhibition } \\
\hline & $r$ & $\begin{array}{c}\text { Partial } \\
r\end{array}$ & $r$ & $\begin{array}{c}\text { Partial } \\
r\end{array}$ & $r$ & $\begin{array}{c}\text { Partial } \\
r\end{array}$ \\
\hline Go Accuracy & -.11 & -.08 & -.14 & -.08 & -.08 & -.04 \\
\hline No-go Accuracy & -.14 & -.11 & $-.21 *$ & -.15 & -.12 & -.08 \\
\hline Go Correct RT & -.10 & -.07 & -.09 & -.03 & .04 & .09 \\
\hline Error RT & -.02 & .00 & -.09 & -.06 & .12 & .15 \\
\hline Go RT variability & -.00 & .01 & .07 & .09 & .16 & $.18^{*}$ \\
\hline Post Error RT & $-.17^{*}$ & -.14 & -.11 & -.04 & .02 & .06 \\
\hline Post Correct Rejection RT & -.09 & -.08 & -.05 & -.01 & .06 & -.01 \\
\hline Post Error Slowing & -.11 & -.09 & -.08 & -.03 & -.05 & -.03 \\
\hline
\end{tabular}

Note. TriPM $=$ Triarchic Psychopathy Measure. Partial $r$ correlations reflect relationships between each individual TriPM scale and each task response measure when controlling for participant gender. $* p<.05$

\subsection{Behavioral effects in the arrow flanker task}

Supplementary Table S5 shows the descriptive statistics for behavioral variables extracted from the arrow flanker task. Split half reliability estimates were high in all cases $(>.85)$, except for the post error slowing effect (.36). Independent samples $t$-test revealed that women participants were more accurate than men on incongruent trials only, responded slower than men on correctly performed trials (with this difference being significant for congruent trials only), and showed greater RT variability for correct responses overall (see Table S5). Regarding within-subjects condition effects, as is typical in the flanker task, participants were less accurate on incongruent trials than on congruent trials, $t(141)=-26.81, p<.0001$, and showed faster correct RTs to congruent stimuli than to incongruent ones, $t(141)=-29.31, p<.0001$. Overall, RTs were slower on correct trials than on error trials, $t(141)=46.82, p<.0001$. Participants also showed significantly slower RTs for correct responses following error trials than 


\section{PERFORMANCE MONITORING ERPS AND TRIARCHIC PSYCHOPATHY - 9} SUPPLEMENT

following correct trials, demonstrating a significant PES effect, $t(141)=7.56, p<.0001$

(see Table S5).

Supplementary Table S5. Split-half Reliabilities, Means (M), and Standard Deviations (SD) for behavioral measures in the arrow flanker task.

\begin{tabular}{|c|c|c|c|c|c|c|c|c|c|}
\hline \multirow[b]{2}{*}{ Flanker task Behavioral Measures } & \multirow[t]{2}{*}{$\begin{array}{l}\text { Split-half } \\
\text { Reliability }\end{array}$} & \multicolumn{2}{|c|}{$\begin{array}{l}\text { All Participants } \\
\quad(n=142)\end{array}$} & \multicolumn{2}{|c|}{$\begin{array}{l}\text { Women } \\
(n=101)\end{array}$} & \multicolumn{2}{|c|}{$\begin{array}{c}\text { Men } \\
(n=41)\end{array}$} & \multicolumn{2}{|c|}{$\begin{array}{c}\text { Women vs } \\
\text { Men difference }\end{array}$} \\
\hline & & $M$ & $S D$ & $M$ & $S D$ & $M$ & $S D$ & $t$ & $p$ \\
\hline Congruent Accuracy (\%) & .90 & 95.11 & 3.34 & 94.78 & 3.39 & 95.91 & 3.11 & -1.82 & .070 \\
\hline Incongruent Accuracy (\%) & .85 & 79.56 & 6.90 & 80.75 & 6.53 & 76.61 & 6.97 & 3.36 & .001 \\
\hline Correct Congruent RT (ms) & .98 & 340 & 27 & 345 & 25 & 330 & 29 & 2.98 & .003 \\
\hline Correct Incongruent RT (ms) & .98 & 392 & 36 & 393 & 35 & 387 & 36 & 1.01 & .313 \\
\hline Overall Correct RT (ms) & .98 & 340 & 27 & 366 & 27 & 355 & 31 & 2.09 & .038 \\
\hline Overall Error RT (ms) & .94 & 288 & 31 & 287 & 29 & 289 & 34 & -0.24 & .812 \\
\hline Correct RT variability (ms) & .93 & 75 & 13 & 77 & 12 & 68 & 11 & 3.13 & .002 \\
\hline Correct Post Error RT (ms) & .89 & 370 & 34 & 375 & 34 & 359 & 34 & 2.00 & .047 \\
\hline Correct Post Correct RT (ms) & .98 & 361 & 30 & 364 & 29 & 353 & 32 & 2.49 & .014 \\
\hline Post Error Slowing (ms) & .36 & 9 & 14 & 10 & 15 & 6 & 12 & 1.80 & .074 \\
\hline
\end{tabular}

Note. $\mathrm{RT}=$ Reaction time. $\mathrm{ms}=$ Milliseconds. Independent samples $t$-tests for evaluation of gender differences have $140 d f$. Significant gender differences are in bold font.

Finally, Supplementary Table S6 shows results from correlational analyses exploring relationships between TriPM scale scores and flanker behavioral measures; these analyses did not reveal any significant associations (all $p \mathrm{~s}>.05$; see Table $\mathrm{S} 6$ ).

Supplementary Table S6. Relationships between TriPM scale scores and behavioral measures in the arrow flanker task.

\begin{tabular}{|c|c|c|c|c|c|c|}
\hline \multirow[b]{2}{*}{ Flanker task Behavioral Measures } & \multicolumn{2}{|c|}{ Boldness } & \multicolumn{2}{|c|}{ Meanness } & \multicolumn{2}{|c|}{ Disinhibition } \\
\hline & $r$ & $\begin{array}{c}\text { Partial } \\
r\end{array}$ & $r$ & $\begin{array}{c}\text { Partial } \\
r\end{array}$ & $r$ & $\begin{array}{c}\text { Partial } \\
r\end{array}$ \\
\hline Congruent Accuracy & -.01 & -.04 & .06 & .00 & -.02 & -.06 \\
\hline Incongruent Accuracy & -.14 & -.10 & -.07 & .03 & .05 & .11 \\
\hline Correct Congruent RT & -.06 & -.02 & -.10 & -.01 & -.10 & -.05 \\
\hline Correct Incongruent RT & -.02 & -.00 & -.07 & -.04 & -.13 & -.12 \\
\hline Overall Correct RT & -.04 & -.01 & -.09 & -.03 & -.12 & -.09 \\
\hline Overall Error RT & .03 & .02 & -.05 & -.07 & -.10 & -.11 \\
\hline Correct RT variability & -.06 & .00 & -.09 & -.00 & -.04 & .02 \\
\hline Correct Post Error RT & -.02 & .01 & -.05 & .03 & -.08 & -.04 \\
\hline Correct Post Correct RT & -.05 & -.02 & -.08 & -.03 & -.12 & -.09 \\
\hline Post Error Slowing & .05 & .08 & .06 & .12 & .07 & .11 \\
\hline
\end{tabular}

Note. TriPM $=$ Triarchic Psychopathy Measure. Partial $r$ correlations reflecting relationships between each individual TriPM scale and each task response measure when controlling for participant gender. ${ }^{*} p<.05$ 
PERFORMANCE MONITORING ERPS AND TRIARCHIC PSYCHOPATHY SUPPLEMENT

\subsection{Stimulus-locked ERP effects: Response inhibition and flanker interference}

Supplementary Table S7 shows descriptive statistics for the stimulus-locked ERPs extracted from the go/no-go and the arrow flanker tasks. Reliabilities were high in all cases $(>.90)$. Independent samples $t$-tests revealed that male participants showed higher P3 amplitudes than women in all conditions of both tasks (i.e., go and no-go - for the go/no-go task; congruent and incongruent - for the flanker task), and also showed significantly diminished (i.e., less negative) N2 amplitudes in the flanker task for congruent trials only (See Table S7).

Supplementary Table S7. Split-half Reliabilities, Means (M), and Standard Deviations (SD) for Stimulus-locked ERPs in the go/no-go and the arrow flanker task.

\begin{tabular}{|c|c|c|c|c|c|c|c|c|c|}
\hline \multirow[b]{2}{*}{ Stimulus-locked ERPs } & \multirow[t]{2}{*}{$\begin{array}{l}\text { Split-half } \\
\text { Reliability }\end{array}$} & \multicolumn{2}{|c|}{$\begin{array}{l}\text { All Participants } \\
\quad(n=142)\end{array}$} & \multicolumn{2}{|c|}{$\begin{array}{l}\text { Women } \\
(n=101)\end{array}$} & \multicolumn{2}{|c|}{$\begin{array}{c}\text { Men } \\
(n=41)\end{array}$} & \multicolumn{2}{|c|}{$\begin{array}{c}\text { Women vs } \\
\text { Men difference }\end{array}$} \\
\hline & & $M$ & $S D$ & $M$ & $S D$ & $M$ & $S D$ & $t$ & $p$ \\
\hline \multicolumn{10}{|l|}{ Go/no-go task } \\
\hline No-go N2 $(\mu \mathrm{V})$ & .90 & -0.27 & 1.06 & -0.33 & 1.01 & -0.11 & 1.17 & -1.12 & .264 \\
\hline Go N2 $(\mu \mathrm{V})$ & .98 & 0.28 & 1.01 & 0.19 & 0.90 & 0.51 & 1.24 & -1.72 & .087 \\
\hline No-go P3 $(\mu \mathrm{V})$ & .94 & 4.07 & 2.78 & 3.22 & 2.26 & 6.14 & 2.76 & -6.52 & .000 \\
\hline Go P3 $(\mu \mathrm{V})$ & .98 & 1.92 & 1.31 & 1.52 & 1.07 & 2.90 & 1.35 & -6.41 & .000 \\
\hline \multicolumn{10}{|l|}{ Arrow flanker task } \\
\hline Incongruent $\mathrm{N} 2(\mu \mathrm{V})$ & .95 & 0.22 & 1.01 & 0.21 & 0.96 & 0.23 & 1.14 & -.09 & .929 \\
\hline Congruent N2 $(\mu \mathrm{V})$ & .94 & 0.62 & 1.17 & 0.48 & 1.10 & 0.98 & 1.27 & -2.33 & .021 \\
\hline Incongruent $\mathrm{P} 3(\mu \mathrm{V})$ & .98 & 2.60 & 1.92 & 2.08 & 1.61 & 3.87 & 2.05 & -5.51 & .000 \\
\hline Congruent $\mathrm{P} 3(\mu \mathrm{V})$ & .98 & 2.52 & 1.84 & 2.04 & 1.55 & 3.69 & 2.00 & -5.28 & .000 \\
\hline
\end{tabular}

Note. $\mu \mathrm{V}=$ Microvolts. Independent samples $t$-tests for evaluation of gender differences have $140 d f$. Significant gender differences are shown in bold font.

Regarding within-subject condition effects, as is typically the case in go/no-go tasks (see Huster, Enriquez-Geppert, Lavallee, Falkenstein \& Herrman, 2013, for a review), a pattern of differential brain activation for the no-go condition relative to the go condition was found for both the N2 and the P3 components. Larger N2 amplitudes were observed for successfully inhibited responses to no-go stimuli (no-go N2) than for correctly responded go stimuli (go N2) at frontocentral-cluster sites, $t(141)=-7.69, p<$ 
PERFORMANCE MONITORING ERPS AND TRIARCHIC PSYCHOPATHY 1 SUPPLEMENT

.0001 . This effect is illustrated in Figure 1.A of the main article. Likewise, P3 amplitudes for the centroparietal electrode cluster were larger for successfully inhibited no-go stimuli (no-go P3), than for correctly responded go stimuli (go P3), $t$ (141) = 14.33, $p<.0001$ (see Figure 1.E of the main article and Table S7 for descriptive statistics).

In the arrow flanker task, and also consistent with previous research (see Larson, Clayson, \& Clawson, 2014, for a review), N2 amplitudes were (relatively) more negative following incongruent stimuli than following congruent stimuli at frontocentral-cluster sites, $t(141)=-6.78, p<.0001$. This effect is illustrated in Figure 1.C of the main article. We did not find a significant difference in P3 amplitude following incongruent stimuli as compared to congruent stimuli at centroparietal sites, $t$ $(141)=1.53, p=.13$ (see Table S7). This effect is illustrated in Figure 1.G of the main article. Visual inspection of these grand-average waveforms suggested that differences between conditions were related to component latencies -with longer latencies for incongruent than for congruent trials- rather than to amplitudes, consistent with other studies examining the P300 component in a similar version of the arrow flanker task (e.g., Hajcak, Moser, Yeung, \& Simons, 2005).

As suggested by an anonymous reviewer, and based on some evidence demonstrating stronger inhibitory effects on the P3 response at frontocentral electrode sites in inhibitory paradigms (e.g., Huster et al., 2013, for a review), along with some studies reporting stronger disinhibition-related reductions of the $\mathrm{P} 3$ response at frontal electrode sites (e.g., Nelson, Patrick, \& Bernat, 2011), we conducted a supplemental analysis testing for differences across regions (frontocentral vs centroparietal) in the go/no-go condition effect for stimulus-locked P3 response, and in the effect for TriPM Disinhibition. Results from a 2 (region: Frontal-Central [FC], Central-Parietal [CP]) x 2 
SUPPLEMENT

(condition: go, no-go) repeated-measures ANOVA revealed significant main effects for both region $\left(F[1,141]=158.22, p<.0001, \eta^{2} \mathrm{p}=0.53 ; \mathrm{CP}: M=2.99 \mu \mathrm{V}, \mathrm{SE}=0.16\right.$, FC: $M=1.22 \mu \mathrm{V}, \mathrm{SE}=0.11)$ and condition $\left(F[1,141]=216.47, p<.0001, \eta^{2}{ }_{\mathrm{p}}=0.61\right.$; no-go: $M=2.93 \mu \mathrm{V}, \mathrm{SE}=0.17$, go: $M=1.28 \mu \mathrm{V}, \mathrm{SE}=0.08$ ), along with a region $\mathrm{x}$ condition interaction $\left(F[1,141]=70.06, p<.0001, \eta^{2}=0.33\right)$. Follow-up paired samples $t$-test revealed that the no-go - go difference was indeed greater at the $\mathrm{CP}$ region $(M=2.15 \mu \mathrm{V}, \mathrm{SD}=1.78 ; t[141]=14.33, p<.0001, d=1.21)$ that at the $\mathrm{FC}$ region $(M=1.16 \mu \mathrm{V}, \mathrm{SD}=1.78 ; t[141]=11.74, p<.0001, d=1.02)$. Partial correlations for TriPM Disinhibition scores with no-go P3 at frontocentral and centroparietal sites, controlling for participant gender, revealed similar relationships for the two regions (partial $r s=-.17$ and $-.18, p s=.050$ and .031 , respectively). Overall, these results provide support for our selection of the centroparietal region for measuring the no-go P3 component reported in the main article, given the stronger effect for the inhibition manipulation found at this region and the similarity in associations with triarchic disinhibition for the two regions.

For completeness, the same analysis was run for the $\mathrm{P} 3$ response in the arrow flanker task, and it revealed a significant main effect of region $(F[1,141]=142.053, p$ $\left.<.0001, \eta_{\mathrm{p}}^{2}=0.50 ; \mathrm{CP}: M=2.56 \mu \mathrm{V}, \mathrm{SE}=0.16, \mathrm{FC}: M=0.95 \mu \mathrm{V}, \mathrm{SE}=0.09\right)$, and a smaller but significant main effect of condition $\left(F[1,141]=8.54, p=.004, \eta_{\mathrm{p}}^{2}=0.06\right.$; incongruent: $M=1.81 \mu \mathrm{V}, \mathrm{SE}=0.11$, congruent: $M=1.70 \mu \mathrm{V}, \mathrm{SE}=0.11)$. However, the region $\mathrm{x}$ condition interaction was not significant $(F[1,141]=1.13, p=0.29)$. As for the no-go P3, results pertaining to associations between incongruent P3 amplitude and TriPM Disinhibition scores - considered in the main article - were slightly stronger at the centroparietal region (partial $r$ controlling for gender $=-.26, p=.002$ ) than at the frontocentral region (partial $r=-.21, p=.011$ ). 

SUPPLEMENT

Finally, Supplementary Table S8 shows results from exploratory correlational analyses between TriPM scale scores and the above-mentioned stimulus-locked ERPs in each task. Neither TriPM Boldness nor Meanness evidenced a significant correlation with any variant of the $\mathrm{N} 2$ or the $\mathrm{P} 3$ in either task (all $p \mathrm{~s}>.05$; see Table S8). As reported in the main article, TriPM Disinhibition scores were inversely related to no-go P3 and incongruent P3 amplitudes after controlling for gender (see Table S8). TriPM Disinhibition scores did not relate significantly to go P3 amplitude, but showed a significant inverse relationship with congruent P3 amplitude, similar to that found for the incongruent P3. This finding is also apparent in Figure 2.C of the main article.

Supplementary Table S8. Relationships between TriPM scale scores and Stimulus-Locked ERPs in both the go/no-go and the arrow flanker task.

\begin{tabular}{|c|c|c|c|c|c|c|}
\hline \multirow[b]{2}{*}{ Stimulus-Locked ERPs } & \multicolumn{2}{|c|}{ Boldness } & \multicolumn{2}{|c|}{ Meanness } & \multicolumn{2}{|c|}{ Disinhibition } \\
\hline & $r$ & $\begin{array}{c}\text { Partial } \\
r\end{array}$ & $r$ & $\begin{array}{c}\text { Partial } \\
r\end{array}$ & $r$ & $\begin{array}{c}\text { Partial } \\
r\end{array}$ \\
\hline \multicolumn{7}{|l|}{ Go/no-go task } \\
\hline No-go N2 & -.00 & -.02 & .14 & .12 & .13 & .12 \\
\hline Go N2 & .05 & .03 & .08 & .03 & .05 & .03 \\
\hline No-go P3 & .02 & -.07 & .11 & -.08 & -.05 & $-.18 *$ \\
\hline Go P3 & .00 & -.10 & .12 & -.05 & -.01 & -.13 \\
\hline \multicolumn{7}{|l|}{ Arrow flanker task } \\
\hline Incongruent $\mathrm{N} 2$ & -.03 & -.03 & -.11 & -.12 & -.07 & -.07 \\
\hline Congruent N2 & -.02 & -.06 & .08 & .01 & .01 & -.03 \\
\hline Incongruent $\mathrm{P} 3$ & -.05 & -.13 & .08 & -.09 & -.14 & $-.26^{* *}$ \\
\hline Congruent P3 & -.03 & -.11 & .09 & -.06 & -.13 & $-.25 * *$ \\
\hline
\end{tabular}

Note. TriPM $=$ Triarchic Psychopathy Measure. Partial $r$ correlations reflect relationships between each individual TriPM scale and each task response measure controlling for participant gender. $* p<.05 ; * * p<.01$.

\subsection{Response-locked ERP effects: Error processing in the go/no-go and the arrow flanker Task}

Supplementary Table S9 shows descriptive statistics for the response-locked ERPs extracted from the go/no-go and arrow flanker tasks. Reliabilities were high in all cases $(>.82)$. Independent samples $t$-tests revealed that male participants showed 
SUPPLEMENT

diminished (less negative) CRN amplitude, as well as greater error-P3 (Pe) and correctresponse Pc amplitudes than women in both tasks, while they did not differ in ERN response amplitude in either task (see Table S9).

Supplementary Table S9. Split-half Reliabilities, Means (M), and Standard Deviations (SD) for Response-locked ERPs in the go/no-go and the arrow flanker task.

\begin{tabular}{|c|c|c|c|c|c|c|c|c|c|}
\hline \multirow[b]{2}{*}{ Response-locked ERPs } & \multirow[t]{2}{*}{$\begin{array}{l}\text { Split-half } \\
\text { Reliability }\end{array}$} & \multicolumn{2}{|c|}{$\begin{array}{l}\text { All Participants } \\
\quad(n=142)\end{array}$} & \multicolumn{2}{|c|}{$\begin{array}{l}\text { Women } \\
(n=101)\end{array}$} & \multicolumn{2}{|c|}{$\begin{array}{c}\text { Men } \\
(n=41)\end{array}$} & \multicolumn{2}{|c|}{$\begin{array}{c}\text { Women vs } \\
\text { Men difference }\end{array}$} \\
\hline & & $M$ & $S D$ & $M$ & $S D$ & $M$ & $S D$ & $t$ & $p$ \\
\hline \multicolumn{10}{|l|}{ Go/no-go task } \\
\hline No-go ERN $(\mu \mathrm{V})$ & .91 & -0.91 & 1.31 & -0.93 & 1.20 & -0.87 & 1.57 & -0.25 & .801 \\
\hline Go CRN $(\mu \mathrm{V})$ & .99 & 0.61 & 1.04 & 0.48 & 0.96 & 0.92 & 1.15 & -2.32 & .022 \\
\hline No-go $\mathrm{Pe}(\mu \mathrm{V})$ & .89 & 4.33 & 2.59 & 3.77 & 2.40 & 5.74 & 2.53 & -4.36 & .000 \\
\hline Go Pc $(\mu \mathrm{V})$ & .95 & 0.76 & 0.88 & 0.63 & 0.74 & 1.10 & 1.09 & -2.96 & .004 \\
\hline \multicolumn{10}{|l|}{ Arrow flanker task } \\
\hline Flanker ERN $(\mu \mathrm{V})$ & .82 & -0.25 & 1.23 & -0.25 & 1.19 & -0.25 & 1.33 & -0.00 & .996 \\
\hline Flanker CRN $(\mu \mathrm{V})$ & .97 & 1.55 & 1.38 & 1.35 & 1.20 & 2.04 & 1.65 & -2.75 & .007 \\
\hline Flanker Pe $(\mu \mathrm{V})$ & .94 & 3.32 & 2.20 & 2.81 & 1.89 & 4.58 & 2.43 & -4.65 & .000 \\
\hline Flanker Pc $(\mu \mathrm{V})$ & .98 & 0.13 & 1.27 & -0.04 & 1.08 & 0.55 & 1.58 & -2.58 & .011 \\
\hline
\end{tabular}

Note. $\mu \mathrm{V}=$ Microvolts. Independent samples $t$-tests for evaluation of gender differences have $140 d f$. Significant gender differences are shown in bold font.

Regarding within-subject condition effects, a pattern of differential brain activation for error response trials relative to correct response trials was found for both the ERN (CRN) and the Pe (Pc) in each task, corroborating past research findings for both components (see Gehring, Liu, Orr, \& Carp, 2012; Overveek, Nieuwenhuis, \& Ridderinkhof, 2005, for reviews). In the go/no-go task, erroneous responses elicited a greater negative deflection (no-go ERN) than correct responses (go CRN) over the frontocentral electrode sites (see Figure 1.I of the main article), $t(141)=-12.58, p<$ .0001. A greater positivity following errors (no-go Pe) as compared to correct responses (go Pc) was observed over centroparietal electrode sites (see Figure 1.M of the main article), $t(141)=18.43, p<.0001$ (see Table S9). Similarly, in the arrow flanker task, error responses (flanker ERN) elicited more negative amplitude responses than correct responses (flanker CRN) over frontocentral electrode sites, $t(141)=-15.94, p<.0001$ 

SUPPLEMENT

(see Figure 1.K in the main article). Finally, a larger centroparietal positivity was observed following error responses (flanker Pe) as compared to correct responses (flanker Pc), $t(141)=18.84, p<.0001$ (see Table S9). This effect is illustrated in Figure 1.0 of the main article.

Supplementary Table S10 shows results from exploratory correlational analyses examining associations of TriPM scale scores with the above mentioned responselocked ERPs in both tasks. Congruent with results reported in the main article, TriPM Disinhibition was associated with diminished no-go ERN amplitudes, as well as to reduced amplitudes for both variants of the Pe response (no-go and flanker), when controlling for gender. Associations for TriPM Boldness and Meanness with these ERPs were non-significant (all $p \mathrm{~s}>.05$; see Table S10). A significant inverse relationship was evident between TriPM Disinhibition and CRN response in the flanker task (more negative CRN amplitudes in participants scoring high in disinhibition), see Table S10.

Supplementary Table S10. Relationships between TriPM scale scores and Response-Locked ERPs in both the go/no-go and the arrow flanker task.

\begin{tabular}{|c|c|c|c|c|c|c|}
\hline \multirow[b]{2}{*}{ Response-Locked ERPs } & \multicolumn{2}{|c|}{ Boldness } & \multicolumn{2}{|c|}{ Meanness } & \multicolumn{2}{|c|}{ Disinhibition } \\
\hline & $r$ & $\begin{array}{c}\text { Partial } \\
r\end{array}$ & $r$ & $\begin{array}{c}\text { Partial } \\
r\end{array}$ & $r$ & $\begin{array}{c}\text { Partial } \\
r\end{array}$ \\
\hline \multicolumn{7}{|l|}{ Go/no-go task } \\
\hline No-go ERN & .08 & .08 & .10 & .10 & $.19^{*}$ & $.19 *$ \\
\hline Go CRN & .08 & .05 & .10 & .04 & .03 & -.01 \\
\hline No-go Pe & -.05 & -.12 & .05 & -.08 & -.09 & $-.18^{*}$ \\
\hline Go Pc & .07 & .03 & .13 & .05 & .06 & .01 \\
\hline \multicolumn{7}{|l|}{ Arrow flanker task } \\
\hline Flanker ERN & .02 & .02 & .00 & .00 & -.05 & -.05 \\
\hline Flanker CRN & .02 & -.02 & .00 & -.08 & $-.22 * *$ & $-.28 * *$ \\
\hline Flanker Pe & -.07 & -.15 & .06 & -.08 & -.12 & $-.22 * *$ \\
\hline Flanker Pc & .00 & -.04 & .07 & .00 & -.04 & -.09 \\
\hline
\end{tabular}

Note. TriPM $=$ Triarchic Psychopathy Measure. Partial $r$ correlations show relationships between each individual TriPM scale and each task response measure controlling for participant gender. $* p<.05 ; * * p<.01$. 

SUPPLEMENT

Visual inspection of Figure 2.G of the main article suggested that this relationship was largely attributable to differences in the preceding positivity on correct trials. Indeed, when computed using a peak-to-peak approach - i.e., CRN peak amplitude minus the most positive peak in the time window from $-75 \mathrm{~ms}$ to $+25 \mathrm{~ms}$ around participant responses - CRN amplitude was no longer associated with TriPM Disinhibition scores $(r=-.06, p=.45$; partial $r$ controlling for gender $=-.03, p=.73)$, nor with TriPM Meanness or Boldness scores $(r \mathrm{~s}<|.05|, p \mathrm{~s}>.53$; partial $r$ s controlling for gender $<|.11|, p \mathrm{~s}>.20)^{1}$.

\section{Exploratory investigation of associations between Triarchic dimensions and difference scores for study ERP measures.}

A series of additional exploratory analyses were conducted to examine associations of condition-difference scores for ERPs from each task with the triarchic trait dimensions. Subtraction-based difference scores were computed to isolate activity specific to: (a) error processing, using the correct response condition as a baseline in both tasks (i.e., ERN minus CRN amplitude, and Pe minus Pc amplitude); (b) inhibitory processing in the go/no-go task (no-go minus go trial amplitude for the N2 and P3 components); and (c) interference control in the flanker task (incongruent minus congruent trial amplitude for the N2). We did not compute incongruent minus congruent P3 amplitude given that we did not find reliable condition differences for P3 response in the arrow flanker task (see section 3.3. above; Figure 1.G of the main article). Results

\footnotetext{
${ }^{1}$ As reported in the main article, we also examined associations between TriPM total scores and the additional behavioral and ERP measures reported here. With respect to task-behavioral measures, TriPM total scores were associated at the bivariate level only with reduced no-go accuracy $(r=-.22, p=.008)$. In partial correlations controlling for gender, this association with no-go accuracy fell below significance (partial $r=-.16, p=.053$ ), but significant negative associations emerged for TriPM total scores with Congruent P3 (partial $r=-.21, p=.011$ ) and CRN (partial $r=-.19, p=.022$ ) amplitudes from the flanker task.
} 
PERFORMANCE MONITORING ERPS AND TRIARCHIC PSYCHOPATHY SUPPLEMENT

from analyses examining correlations for these measures with TriPM scale scores are shown in Supplementary Table S11.

\begin{tabular}{|c|c|c|c|c|c|c|}
\hline \multirow[b]{2}{*}{ Difference scores } & \multicolumn{2}{|c|}{ Boldness } & \multicolumn{2}{|c|}{ Meanness } & \multicolumn{2}{|c|}{ Disinhibition } \\
\hline & $r$ & $\begin{array}{c}\text { Partial } \\
r\end{array}$ & $r$ & $\begin{array}{c}\text { Partial } \\
r\end{array}$ & $r$ & $\begin{array}{c}\text { Partial } \\
r\end{array}$ \\
\hline \multicolumn{7}{|l|}{ Response inhibition } \\
\hline No-go - Go N2 & -.06 & -.06 & .08 & .11 & .10 & .11 \\
\hline No-go - Go P3 & .03 & -.04 & .07 & -.08 & -.07 & $-.18^{*}$ \\
\hline \multicolumn{7}{|l|}{ Flanker Interference } \\
\hline Incongruent - Congruent N2 & -.02 & .03 & $-.25^{* *}$ & $-.17^{*}$ & -.11 & -.05 \\
\hline \multicolumn{7}{|l|}{ Error processing } \\
\hline No-go ERN - Go CRN & .02 & .04 & .02 & .07 & .16 & $.19 *$ \\
\hline No-go Pe - Go Pc & -.08 & -.14 & .01 & -.11 & -.13 & $-.20 *$ \\
\hline Flanker ERN - Flanker CRN & -.00 & .04 & -.00 & .09 & $.18^{*}$ & $.24 * *$ \\
\hline Flanker Pe - Flanker Pc & -.08 & -.13 & .02 & -.08 & -.11 & $-.17 *$ \\
\hline
\end{tabular}

Note. TriPM $=$ Triarchic Psychopathy Measure. Partial $r$ correlations show relationships between each individual TriPM scale and each task response measure controlling for participant gender. ${ }^{*} p<.05 ; * *<.01$.

Results from this set of analysis generally mirrored those reported for absolute ERP amplitude scores in the main article, with only two exceptions. First, TriPM Meanness scores were related to greater ERP flanker N2 differentiation between incongruent and congruent amplitudes (see Table S11). However, this effect was qualified by contrasting (albeit non-significant) associations for this scale with incongruent $\mathrm{N} 2(r=-.11)$ and congruent $\mathrm{N} 2(r=.08) \mathrm{N} 2$ amplitudes (see Table S8). Second, a significant positive association between TriPM Disinhibition and flanker ERN difference amplitude (indicative of diminished differentiation) was also found, but contrary to the corresponding effect for the ERN difference in the go/no-go task which was driven by reduced no-go ERN, but not CRN amplitudes (see Table S10) the relationship with flanker ERN difference was driven by a significant negative 
PERFORMANCE MONITORING ERPS AND TRIARCHIC PSYCHOPATHY SUPPLEMENT

association between TriPM Disinhibition and flanker CRN amplitudes (potentially explained by differences in the preceding positivity to the CRN in the flanker task, as discussed above in section 3.4). Finally, and congruent with the reported reductions of no-go P3/Pe amplitudes and flanker Pe amplitude in highly disinhibited participants (see Tables S8 and S10), TriPM Disinhibition scores showed significant negative associations with no-go minus go Pe and no-go minus go P3 difference scores, as well as with flanker Pe minus flanker Pc difference scores, when controlling for gender (see Table S11). 
PERFORMANCE MONITORING ERPS AND TRIARCHIC PSYCHOPATHY 1 SUPPLEMENT

\section{Supplementary References}

Almeida, P. R., Seixas, M. J., Ferreira-Santos, F., Vieira, J. B., Paiva, T. O., Moreira, P. S., \& Costa, P. (2015). Empathic, moral and antisocial outcomes associated with distinct components of psychopathy in healthy individuals: A Triarchic model approach. Personality and Individual Differences, 85, 205-211. https://doi.org/10.1016/j.paid.2015.05.012

Costa, P. T., \& McCrae, R. R. (1992). Professional manual: Revised NEO Personality Inventory (NEO-PI-R) and NEO Five-Factor Inventory (NEO-FFI). Odessa, FL: Psychological Assessment Resources.

Esteller, À., Poy, R., \& Moltó, J. (2016). Deficient aversive-potentiated startle and the Triarchic model of psychopathy: The role of boldness. Biological Psychology, 117, 131-140. https://doi.org/10.1016/j.biopsycho.2016.03.012

Gehring, W. J., Liu, Y., Orr, J. M., \& Carp, J. (2012). The error-related negativity (ERN/Ne). In S. J. Luck and E. S. Kappenman (Eds.), The Oxford Handbook of Event-related Potential Components (pp. 231-300). New York: Oxford University Press. https://doi.org/10.1093/oxfordhb/9780195374148.013.0120

Hajcak, G., Moser, J. S., Yeung, N., \& Simons, R. F. (2005). On the ERN and the significance of errors. Psychophysiology, 42(2), 151-160. https://doi.org/10.1111/j.1469-8986.2005.00270.x

Huster, R. J., Enriquez-Geppert, S., Lavallee, C. F., Falkenstein, M., \& Herrmann, C. S. (2013). Electroencephalography of response inhibition tasks: Functional networks and cognitive contributions. International Journal of Psychophysiology, 87(3), 217-233. https://doi.org/10.1016/j.ijpsycho.2012.08.001 
PERFORMANCE MONITORING ERPS AND TRIARCHIC PSYCHOPATHY -

SUPPLEMENT

Larson, M. J., Clayson, P. E., \& Clawson, A. (2014). Making sense of all the conflict: a theoretical review and critique of conflict-related ERPs. International Journal of Psychophysiology, 93(3), 283-297. https://doi.org/10.1016/j.ijpsycho.2014.06.007

Nelson, L. D., Patrick, C. J., \& Bernat, E. M. (2011). Operationalizing proneness to externalizing psychopathology as a multivariate psychophysiological phenotype. Psychophysiology, 48(1), 64-72. https://doi.org/10.1111/j.1469$\underline{8986.2010 .01047 . \mathrm{x}}$

Overbeek, T. J., Nieuwenhuis, S., \& Ridderinkhof, K. R. (2005). Dissociable components of error processing: On the functional significance of the Pe vis-à-vis the ERN/Ne. Journal of Psychophysiology, 19(4), 319-329. https://doi.org/10.1027/0269$\underline{8803.19 .4 .319}$

Patrick, C. J., \& Drislane, L. E. (2015). Triarchic model of psychopathy: Origins, operationalizations, and observed linkages with personality and general psychopathology. Journal of Personality, 83(6), 627-643. https://doi.org/10.1111/jopy.12119

Patrick, C. J., Fowles, D. C., \& Krueger, R. F. (2009). Triarchic conceptualization of psychopathy: Developmental origins of disinhibition, boldness, and meanness. Development and Psychopathology, 21(3), 913-938. https://doi.org/10.1017/S0954579409000492

Poy, R., Segarra, P., Esteller, À., López, R., \& Moltó, J. (2014). FFM description of the triarchic conceptualization of psychopathy in men and women. Psychological Assessment, 26(1), 69-76. https://doi.org/10.1037/a0034642

Patton, J. H., Stanford, M. S., \& Barratt, E. S. (1995). Factor structure of the Barratt impulsiveness scale. Journal of Clinical Psychology, 51(6), 768-774. 
PERFORMANCE MONITORING ERPS AND TRIARCHIC PSYCHOPATHY -

SUPPLEMENT

https://doi.org/10.1002/1097-4679(199511)51:6<768::AID-

$\underline{\mathrm{JCLP} 2270510607>3.0 . C O ; 2-1}$

Rabbitt, P. M. (1966). Errors and error correction in choice-response tasks. Journal of Experimental Psychology, 71(2), 264-272. https://doi.org/10.1037/h0022853

Sellbom, M., \& Phillips, T. R. (2013). An examination of the triarchic conceptualization of psychopathy in incarcerated and nonincarcerated samples. Journal of Abnormal Psychology, 122(1), 208-214. https://doi.org/10.1037/a0029306

Sica, C., Drislane, L., Caudek, C., Angrilli, A., Bottesi, G., Cerea, S., \& Ghisi, M. (2015). A test of the construct validity of the Triarchic Psychopathy Measure in an Italian community sample. Personality and Individual Differences, 82, 163-168. https://doi.org/10.1016/j.paid.2015.03.015

Spielberger, C.D., Gorsuch, R.L., \& Lushene, R.E. (1970). The State-Trait Anxiety Inventory: Test manual. Palo Alto, CA: Consulting Psychologist Press.

Stanley, J. H., Wygant, D. B., \& Sellbom, M. (2013). Elaborating on the construct validity of the Triarchic Psychopathy Measure in a criminal offender sample. Journal of Personality Assessment, 95(4), 343-350.

https://doi.org/10.1080/00223891.2012.735302

Strickland, C. M., Drislane, L. E., Lucy, M., Krueger, R. F., \& Patrick, C. J. (2013). Characterizing psychopathy using DSM-5 personality traits. Assessment, 20(3), 327-338. https://doi.org/10.1177/1073191113486691

Torrubia, R., Ávila, C., Moltó, J., \& Caseras, X. (2001). The Sensitivity to Punishment and Sensitivity to Reward Questionnaire (SPSRQ) as a measure of Gray's anxiety and impulsivity dimensions. Personality and individual differences, 31(6), 837862. https://doi.org/10.1016/S0191-8869(00)00183-5 
PERFORMANCE MONITORING ERPS AND TRIARCHIC PSYCHOPATHY -

SUPPLEMENT

van Dongen, J. D., Drislane, L. E., Nijman, H., Soe-Agnie, S. E., \& van Marle, H. J.

(2017). Further evidence for reliability and validity of the triarchic psychopathy measure in a forensic sample and a community sample. Journal of Psychopathology and Behavioral Assessment, 39(1), 58-66.

https://doi.org/10.1007/s10862-016-9567-5 